arXiv:0805.3931

$\mathrm{KEK}-\mathrm{TH}-1253$

DESY 08-062

\title{
The FZZ-Duality Conjecture - A Proof
}

\author{
Yasuaki Hikida ${ }^{1}$ and Volker Schomerus ${ }^{2}$ \\ ${ }^{1}$ High Energy Accelerator Research Organization (KEK) \\ Tukuba, Ibaraki 305-0801, Japan \\ ${ }^{2}$ DESY Theory Group, DESY Hamburg \\ Notkestrasse 85, D-22603 Hamburg, Germany
}

May 2008

\begin{abstract}
We prove that the cigar conformal field theory is dual to the Sine-Liouville model, as conjectured originally by Fateev, Zamolodchikov and Zamolodchikov. Since both models possess the same chiral algebra, our task is to show that correlations of all tachyon vertex operators agree. We accomplish this goal through an off-critical version of the geometric Langlands duality for $\mathrm{sl}(2)$. More explicitly, we combine the well-known self-duality of Liouville theory with an intriguing correspondence between the cigar and Liouville field theory. The latter is derived through a path integral treatment. After a very detailed discussion of genus zero amplitudes, we extend the duality to arbitrary closed surfaces.
\end{abstract}

e-mail: hikida@post.kek.jp, volker.schomerus@desy.de 


\section{Contents}

1 Introduction $\quad 2$

2 The $H_{3}^{+}$-Liouville correspondence $\quad 5$

2.1 Correlators in the $H_{3}^{+}$WZNW model . . . . . . . . . . . . . . . . . . 6

2.2 The correspondence with Liouville theory . . . . . . . . . . . . . . . . 8

3 The Cigar-Sine-Liouville duality $\quad 11$

3.1 A correspondence between the cigar and Liouville theory . . . . . . . . . . 11

3.2 Derivation of the duality with Sine-Liouville theory . . . . . . . . . . . . 14

3.2.1 A weak-strong coupling correspondence . . . . . . . . . . . . . . 14

3.2.2 Removing degenerate field insertions . . . . . . . . . . . . . . 15

3.2.3 Relation with Sine-Liouville theory . . . . . . . . . . . . . . 17

4 Generalization to surfaces of higher genus $\quad 20$

4.1 The $H_{3}^{+}$- Liouville correspondence - genus $g \geq 1 \ldots$. . . . . . . . . . 20

4.2 The cigar-Liouville correspondence - genus $g \geq 1 \ldots \ldots$. . . . . . . . . 24

5 Conclusion and open problems 25

A Cigar field theory as a gauged WZNW model 28

B Reflection: Self-duality and field identification $\quad 31$

B.1 Field identification in Liouville field theory . . . . . . . . . . . . . . . 31

B.2 Reflection in theories with several bosonic fields . . . . . . . . . . . . 32

C On the Jacobian 33

C.1 The Jacobian on the sphere . . . . . . . . . . . . . . . . . . 33

C.2 The Jacobian for genus $g \geq 1 \ldots \ldots . \ldots \ldots$ 


\section{Introduction}

Strong-weak coupling dualities in 2-dimensional quantum theory possess a long history, with a remarkable range of applications. In addition to providing indispensable tools for concrete calculations, they have also taught us important lessons about non-perturbative quantum field theory in general. Among these dualities, those relating different target space geometries of sigma models have received special attention, in particular from string theorists. Many extensions of the famous $R \leftrightarrow 1 / R$ duality for a compactified free bosonic field $X \sim X+2 \pi R$ were found, see e.g. [1] for a review of developments in string theory and further references to original research papers. Mirror symmetry of Calabi-Yau compactifications has allowed to sum up contributions from world-sheet instantons, a task that would seem virtually impossible without duality symmetries.

Non-perturbative contributions to the $\alpha^{\prime}$ dependence of the world-sheet model are potentially important whenever the target space of the string becomes strongly curved, or, more generally, when some geometric length scale is of the order of the string length $l_{s} \sim \sqrt{\alpha^{\prime}}$. In recent years, a new class of curved target spaces has come into focus. Through the AdS/CFT correspondence, strongly curved (asymptotically) Anti-de Sitter (AdS) backgrounds are believed to encode interesting information about gauge field theories. As simple examples show, the corresponding world-sheet theories receive nonperturbative corrections. It would be of obvious interest to capture those through perturbative expansions in some dual field theory, whenever such a dual exists.

At the moment, very little is known about target space dualities involving AdS or more general holographic backgrounds, with only one exception. It is provided by the 2D Euclidean black hole, also known as the (semi-infinite) cigar. The cigar conformal field theory contains two fundamental fields, $\phi$ and $X$, which take their values in the real line $\phi \in]-\infty, \infty]$ and in the interval $X \in[0,2 \pi R]$, respectively. In these coordinates, the non-trivial background metric $d s^{2}$ and the dilaton $\Phi$ read $[2,3,4]$

$$
d s^{2}=k \frac{e^{-2 \phi}}{1+e^{-2 \phi}}\left(d \phi^{2}+k^{-1} d X^{2}\right), \quad e^{-2 \Phi}=e^{-2 \Phi_{0}}\left(1+e^{-2 \phi}\right) .
$$

The compactification radius $R$ of the field $X$ is related to the parameter $k$ in the metric by $k=R^{2}$. Throughout this entire note we shall set $\alpha^{\prime}=1$. The cigar geometry, along with its Lorentzian counterpart and supersymmetric extensions, possesses many interesting applications. For us, it serves as the simplest holographic background in which duality can be addressed (see e.g. [5] and references therein). 
Duality symmetries in quantum field theory should come with a prescription that maps fields of one model onto those of its dual. Let us recall that the tachyon vertex operators of the cigar conformal field theory are associated with the asymptotic data of wave functions at $\phi=-\infty$,

$$
\Psi_{(n, w)}^{\mathrm{cig} ; j}(z, \bar{z})=e^{2 b(j+1) \phi} e^{i \frac{n}{R} X+i R w \widetilde{X}} \quad \text { where } \quad \widetilde{X}=\widetilde{X}(z, \bar{z})=-i \int^{(z, \bar{z})} * d X
$$

is dual to the field $X=X(z, \bar{z})$. It is defined through a line integral over the Hodge dual $* d X$ of the differential $d X$. We have also introduced the parameter $b=1 / \sqrt{k-2}$ that will appear frequently throughout our entire presentation. The parametrization of tachyon vertex operators in terms of the complex radial momentum $j$ and the quantum numbers $n, w$ for the circle direction follows the usual conventions.

According to a famous FZZ-conjecture of V. Fateev, Al. Zamolodchikov and A.B. Zamolodchikov [6] (see also [5] for a review), a dual of the cigar conformal field theory is given by the so-called Sine-Liouville model. This dual theory also involves two fields $\phi$ and $X$ with values in $\phi \in]-\infty, \infty[$ and $X \in[0,2 \pi R]$, as before. The two coordinates parametrize a cylinder with the trivial flat metric and radius $R=\sqrt{k}$. The motion of strings towards $\phi \sim \infty$ is cut off by a tachyon potential of the form

$$
V(\phi, X)=4 \pi \lambda e^{\frac{1}{b} \phi} \cos (\sqrt{k} \tilde{X})
$$

The operator $V$ is marginal, provided the field $\phi$ has background charge $Q_{\phi}=b$, the same as in the cigar model above. Tachyon vertex operators in Sine-Liouville theory are again parametrized by data in the asymptotic domain $\phi \rightarrow-\infty$ where the interaction is exponentially suppressed, i.e. they take the same form as for the cigar

$$
\Psi_{(n, w)}^{\mathrm{SL} ; j}(z, \bar{z})=e^{2 b(j+1) \phi} e^{i \frac{n}{R} X+i R w \widetilde{X}}
$$

The FZZ conjecture claims that one may identify vertex operators in the cigar background and Sine-Liouville theory,

$$
\left\langle\prod_{\nu=1}^{N} \Psi_{\left(n_{\nu}, w_{\nu}\right)}^{\mathrm{cig} ; j_{\nu}}\left(z_{\nu}, \bar{z}_{\nu}\right)\right\rangle_{(k)}^{\mathrm{cig}}=\mathcal{N}\left\langle\prod_{\nu=1}^{N} \Psi_{\left(w_{\nu}, n_{\nu}\right)}^{\mathrm{SL} ; j_{\nu}}\left(z_{\nu}, \bar{z}_{\nu}\right)\right\rangle_{R}^{\mathrm{SL}} .
$$

The normalization $\mathcal{N}$ depends on the parameter $b$, the winding number violation $S=$ $\sum w_{\nu}$, and the number $N$ of vertex operators. The coupling constant $\lambda$ of Sine-Liouville theory relates to the parameter $b$. An explicit formula will be spelled out below. 
The FZZ-duality has several interesting features. To begin with, it is a strong-weak coupling duality. In fact, the cigar conformal field theory is weakly coupled for large $k$ or, equivalently, for small $b$. In this regime, the tachyon potential (1.3) increases rapidly towards larger values of $\phi$. Hence, the model becomes strongly coupled. Another point worth stressing is that the FZZ-duality relates a sigma model to another 2-dimensional field theory with constant metric and linear dilaton, but non-trivial tachyon potential. The latter is a finite sum of exponentials. In this respect, the relation between the cigar and Sine-Liouville theory is very different from the geometric target space dualities which are produced e.g. by the Buscher rules [7, 8]. Some tests of the FZZ-duality on the sphere were performed in [9]. A supersymmetric version was established in [10], but their argument is passing through gauged linear sigma models and hence rather indirect. Our aim here is to give a direct derivation of the equality (1.5), first for the sphere and then on an arbitrary surface.

Our proof involves two key ingredients. As a starting point, we represent the cigar theory as a coset conformal field theory, obtained by gauging a certain U(1) symmetry in the $H_{3}^{+}$WZNW model. The coset formulation then allows us to invoke an intriguing correspondence between correlation functions in the $H_{3}^{+}$WZNW model and Liouville field theory $[11,12]$. Thereby, we shall be able to express correlation functions of the cigar conformal field theory through special correlators in a product of Liouville field theory and a free boson. The additional bosonic field arises from the gauge field of the coset construction. It participates actively in the reduction from the cigar to Liouville field theory. Up to this point, all steps are performed in the path integral formulation of the cigar, following closely our recent work [12]. Clearly, such path integral manipulations are not sufficient to transfer us from a weakly to a strongly coupled regime. This is where the second central ingredient comes in. Let us recall that Liouville field theory is self-dual, i.e. it looks exactly the same at weak and strong coupling. Having expressed correlators of the cigar through Liouville theory we can capitalize on the self-duality of the latter to describe the cigar in the strong coupling regime. The resulting formulation of the cigar conformal field theory will not look like Sine-Liouville theory at first, but the two descriptions shall turn out to be related through simple rotations and reflections in field space. We shall describe these explicitly in the last part of our derivation, following ideas from an unpublished note of V. Fateev [13].

The plan of our work is as follows. In the next section we shall review and extend 
our previous path integral derivation of the correspondence between the $\mathrm{H}_{3}^{+}$WZNW model and Liouville field theory. The discussion will incorporate sectors obtained by the action of spectral flow [14]. Such an extension was described by Ribault in [15] and it is crucial for us in order to treat winding number violating amplitudes of the cigar conformal field theory. Our proof of eq. (1.5) is then presented in section 3. The fourth section contains a generalization of the FZZ-duality and its proof for higher genus surfaces. In the conclusions we finally present a list of open problems and possible applications.

\section{The $H_{3}^{+}$-Liouville correspondence}

The main purpose of this section is to extend our path integral derivation of the correspondence between the $\mathrm{H}_{3}^{+}$WZNW model and Liouville field theory to sectors obtained through spectral flow [14]. On the sphere, the corresponding relation between correlation functions has been derived by algebraic means in [15]. Generalizing the treatment of [12], we shall arrive at the same result. Our new derivation, however, has two advantages. First of all, it also applies to the case of maximal winding number violation that could not be treated previously [15]. More importantly, our argument extends to surfaces of higher genus. Those will be dealt with in section 4 .

In deducing the main formula (2.17) below, we shall sketch the key ideas explained in [12]. As we are going through the individual steps, we shall present them in a somewhat different light, stressing the similarities with the standard derivation of T-duality. Of course, the correspondence between the $\mathrm{H}_{3}^{+}$model and Liouville field theory is not a T-duality, as e.g. both theories possess different central charges. Nevertheless, through this correspondence, Liouville theory manages to capture all information about tachyon correlators in the $H_{3}^{+}$model. At first sight, this might actually seem a bit surprising. While tachyon vertex operators in the WZNW model carry a 3-component target space momentum, tachyonic modes in Liouville field theory possess momentum in one direction only. The resolution of this apparent paradox is intriguing: Only the radial momentum of the WZNW model is mapped to a momentum in Liouville theory. The two remaining components of target space momentum, on the other hand, become parameters specifying the world-sheet insertion points for degenerate fields in Liouville theory. Such additional insertions are necessary precisely because the models on both sides of the correspondence have different target space dimension (central charge). 


\subsection{Correlators in the $H_{3}^{+}$WZNW model}

As is any derivation of T-dualities (see, e.g. $[7,8]$ ), our first step is to present the $H_{3}^{+}$ WZNW model in a first order formulation. To this end, we introduce two auxiliary fields $\beta$ and $\bar{\beta}$ of weight $h=1$. These supplement three fields $\phi, \gamma$ and $\bar{\gamma}$ of conformal weight $h=0$ that come with the target space coordinates of the Euclidean $A d S_{3}$. The action of the model reads

$$
S_{k}^{\mathrm{WZNW}}[\phi, \gamma, \beta]=\frac{1}{2 \pi} \int d^{2} w\left(\partial \phi \bar{\partial} \phi-\beta \bar{\partial} \gamma-\bar{\beta} \partial \bar{\gamma}+\frac{Q_{\phi}}{4} \sqrt{g} \mathcal{R} \phi-b^{2} \beta \bar{\beta} e^{2 b \phi}\right)
$$

In order for the interaction term to be marginal, the field $\phi$ must possess a background charge $Q_{\phi}=b=1 / \sqrt{k-2}$. The usual WZNW model on $H_{3}^{+}$may be recovered from eq. (2.1) by integration over $\beta$ and $\bar{\beta}$.

Our task is to compute correlation functions of tachyon vertex operators. There exist several natural ways to parametrize the space of tachyonic modes. The choice we shall adopt is given by

$$
V_{j}(\mu \mid z) \equiv|\mu|^{2 j+2} e^{\mu \gamma(z)-\bar{\mu} \bar{\gamma}(\bar{z})} e^{2 b(j+1) \phi(z, \bar{z})}
$$

These vertex operators are labeled directly by the three target space momenta $\mu, \bar{\mu}$ and $j$. Our particular choice of $\mu$-dependent prefactor will turn out to be very convenient later on. The quantities we want to compute are the correlators

$$
\left\langle\prod_{\nu=1}^{N} V_{j_{\nu}}\left(\mu_{\nu} \mid z_{\nu}\right) v^{S}(\xi)\right\rangle^{H}=\int_{(S, \xi)} \mathcal{D} \phi \mathcal{D} \gamma \mathcal{D} \beta e^{-S_{k}^{\mathrm{WZNW}}[\phi, \gamma, \beta]} \prod_{\nu=1}^{N} V_{j_{\nu}}\left(\mu_{\nu} \mid z_{\nu}\right) e^{S \phi(\xi) / b} .
$$

Here, the superscript $H$ reminds us to evaluate the correlation function in the $H_{3}^{+}$WZNW model. The operator $v^{S}(\xi)$ we inserted at $z=\xi$ on the left hand side is obtained by acting with $S$ units of spectral flow on the identity field. We let the superscript $S$ run through positive integers. The generalization to negative $S$ is quite obvious but dealing with both signs at the same time would clutter our presentation below. The insertion of $v^{S}(\xi)$ has two effects on the right hand side of eq. (2.3). To begin with, it leads to an insertion of the vertex operator $\exp (S \phi / b)$. Moreover, $v^{S}(\xi)$ determines the integration domain for the fields $\beta$ and $\bar{\beta}$ in the path integral. To be more precise, the integration in eq. (2.3) is meant to extend over all field configurations such that $\beta$ and $\bar{\beta}$ both possess a zero of order $S$ at $z=\xi$. In our analysis [12], we had set the parameter $S$ to $S=0$ and studied 
the usual path integral for vacuum expectation values. With later applications in mind we now extend this treatment.

Even though we may think of $v^{S}(\xi)$ as being defined through the path integral representation we outlined in the previous paragraph, it might be useful to pause for a moment and explain the precise relation to the action of the spectral flow automorphism $\rho^{S}$ on the affine $\operatorname{sl}(2)$ current algebra. In the following discussion we shall set $\xi=0$ and pass to an operator formalism. Our freedom of choosing $S$ can then be understood as the insertion of a state $|S\rangle$ that is obtained from the vacuum $|0\rangle$ through $S$ units of spectral flow, i.e.

$$
\left\langle\prod_{\nu=1}^{N} V_{j_{\nu}}\left(\mu_{\nu} \mid z_{\nu}\right) v^{S}(0)\right\rangle^{H}=\left\langle 0\left|\prod_{\nu=1}^{N} V_{j_{\nu}}\left(\mu_{\nu} \mid z_{\nu}\right)\right| S\right\rangle,
$$

where $|S\rangle$ satisfies

$$
\rho^{S}\left(J_{n}^{a}\right)|S\rangle=0 \quad \text { for } \quad n \geq 0, a=3, \pm
$$

For the reader's convenience we recall that the spectral flow automorphism $\rho^{S}$ of the sl(2) current algebra is defined by

$$
\rho^{S}\left(J_{n}^{3}\right)=J_{n}^{3}-\frac{k}{2} S \delta_{n, 0} \quad, \quad \rho^{S}\left(J_{n}^{ \pm}\right)=J_{n \pm S}^{ \pm}
$$

We would like to rephrase the conditions (2.4) in terms of the fields that appear in our action. To this end, we spell out the usual free field realization of $\mathrm{sl}(2)$ currents,

$$
\begin{aligned}
& J^{-}(z)=\beta(z), \quad J^{3}(z)=: \beta(z) \gamma(z):-b^{-1} \partial \phi(z) \\
& J^{+}(z)=: \beta(z) \gamma^{2}(z):-2 b^{-1} \gamma(z) \partial \phi(z)+k \partial \gamma(z)
\end{aligned}
$$

The construction of currents through $\beta, \gamma$ and $\phi$ implies that $|S\rangle=|S\rangle_{(\beta, \gamma)} \otimes|S\rangle_{\phi}$ may be characterized through

$$
\beta_{n-S}|S\rangle_{(\beta, \gamma)}=0 \quad, \quad \gamma_{n+S}|S\rangle_{(\beta, \gamma)}=0 \quad \text { for } n \geq 0
$$

Furthermore, the state $|S\rangle_{\phi}$ carries a non-vanishing charge with respect to the zero mode of the field $\phi$, i.e.

$$
|S\rangle_{\phi}=e^{\frac{S}{b} \phi(0)}|0\rangle_{\phi}
$$

By now we easily recognise the description of $v^{S}(\xi)$ we gave above. In fact, the state $|S\rangle$ creates a zero of order $S$ in the field $\beta(w)=\sum \beta_{n} w^{-n-1}$ at $w=0$. The effect of $|S\rangle$ on 
the field $\phi$ is captured by the insertion of the vertex operator $\exp (S \phi / b)$. Obviously, the insertion point of $v^{S}$ can be moved from $w=0$ to any point on the sphere (or surface). The amplitudes (2.3) we considered here are $(N+1)$-point functions containing $N$ unflowed states in addition to the state at $z=\xi$. The latter is obtained from the identity by $S$ units of spectral flow as mentioned before. More general correlators for which the $S$ units are distributed among all $N+1$ fields are rather easy to find, as discussed in [15]. The relation between the cigar and Liouville field theory would be derived from its simplest form when all the spectral flow is assembled in one insertion point.

\subsection{The correspondence with Liouville theory}

Since vertex operators do not depend on $\beta$ and $\bar{\beta}$, these fields can easily be integrated out. The resulting action is that of the WZNW model for the usual coordinate fields $\gamma, \bar{\gamma}$ and $\phi$. A "dual" description of the WZNW model emerges when we integrate out $\gamma$ and $\bar{\gamma}$ instead of $\beta$ and $\bar{\beta}$. As in the case of T-dualities, the integration over $\gamma$ and $\bar{\gamma}$ gives constraints on $\beta$ and $\bar{\beta}$. Solutions to these constraints are inserted back into the action. Thereby, we arrive at the dual formulation we are after. Let us now see how all this works out for our $H_{3}^{+}$WZNW model.

As explained in much detail in [12], integration over $\gamma$ and $\bar{\gamma}$ gives the constraints

$$
\bar{\partial} \beta(w)=2 \pi \sum_{\nu=1}^{N} \mu_{\nu} \delta^{2}\left(w-z_{\nu}\right) \quad, \quad \partial \bar{\beta}(\bar{w})=-2 \pi \sum_{\nu=1}^{N} \bar{\mu}_{\nu} \delta^{2}\left(w-z_{\nu}\right) .
$$

If it were not for the insertion of vertex operators, these would simply require the onedifferentials $\beta$ and $\bar{\beta}$ to be (anti-)holomorphic. The vertex operators act like sources and force $\beta$ and $\bar{\beta}$ to possess first order poles with residues $\mu_{\nu}$ and $-\bar{\mu}_{\nu}$ at the insertion points, respectively. On the complex sphere, a meromorphic one-differential with these properties is uniquely determined to be of the form

$$
\beta(w)=\sum_{\nu=1}^{N} \frac{\mu_{\nu}}{w-z_{\nu}}
$$

and similarly for $\bar{\beta}$. Due to the insertion of $v^{S}(\xi), \beta$ and $\bar{\beta}$ possess a zero of order $S$ at $w=\xi$. Therefore, the parameters $\mu_{\nu}$ must obey the following $S+1$ equations

$$
\sum_{\nu=1}^{N} \frac{\mu_{\nu}}{\left(\xi-z_{\nu}\right)^{n}}=0 \quad \text { for } \quad n=0,1,2, \ldots, S .
$$


The first equation with $n=0$ ensures that $\beta(w)$ has no pole at $w=\infty$. The uniqueness of the solution to eqs. (2.10) is a new feature of our analysis, distinguishing it from the case of usual T-dualities. In the standard cases, solutions to the constraints are parametrized by a dual field. Thereby, field theories related by an ordinary T-duality possess the same number of fields. Because the solutions to our constraint equations (2.10) are unique, the resulting "dual" of the $\mathrm{H}_{3}^{+}$WZNW model will have two fields less than the theory we started with.

The next step is to insert the solutions to the constraints back into the action. This leaves us with a theory of a single field $\phi$ and a Liouville-like interaction term. A second glance at the resulting action, however, reveals an unpleasant feature: In the place of Liouville's cosmological constant we find a rather complicated function $|\beta(w)|^{2}$ depending on the insertion points $z_{\nu}$ and momenta $\mu_{\nu}, \bar{\mu}_{\nu}$. We can try to resolve this issue by absorbing the unwanted function $|\beta(w)|^{2}$ into a shift of the zero mode of $\phi$. Since we are going to shift $\phi$ by the logarithm of $\beta(w) \bar{\beta}(\bar{w})$, it is advantageous to bring $\beta(w)$ into a product form first. Let us recall that for any one-differential, the number of poles exceeds the number of zeroes by two. Hence, $\beta(w)$ must have $N-2$ zeroes. Since we inserted the operator $v^{S}(\xi), S$ of these zeroes must come together at $w=\xi$. We will denote the remaining $N-2-S$ locations of zeroes on the sphere by $w=y_{i}{ }^{1}$ Furthermore, a differential is uniquely characterized by the position of its zeroes and poles up to an overall factor $u$. Consequently, we can rewrite $\beta(w)$ in the form

$$
\beta(w)=u \frac{(w-\xi)^{S} \prod_{i=1}^{N-2-S}\left(w-y_{i}\right)}{\prod_{\nu=1}^{N}\left(w-z_{\nu}\right)}=: u \mathcal{B}(w)
$$

Thereby, we have now replaced the $N$ parameters $\mu_{\nu}$ subject to constraints (2.12) through $N-2-S$ coordinates $y_{i}$ and a global factor $u$. Now we are ready to introduce the new bosonic field $\varphi$ through

$$
\varphi:=\phi+\frac{1}{2 b}\left(S \ln |w-\xi|^{2}+\sum_{i=1}^{N-2-S} \ln \left|w-y_{i}\right|^{2}-\sum_{\nu=1}^{N} \ln \left|w-z_{\nu}\right|^{2}-\ln |u \rho(w)|^{2}\right)
$$

where the term in brackets is $\ln |u \mathcal{B}|^{2}$. Here we have included a non-trivial Weyl factor $\rho(z)$. With this factor, the world-sheet metric and its curvature are given as $d s^{2}=$ $|\rho(z)|^{2} d z d \bar{z}$ and $\sqrt{g} \mathcal{R}=-4 \partial \bar{\partial} \ln |\rho|$. Throughout most of the present note we set $\rho(z)=1$.

\footnotetext{
${ }^{1}$ In this way we have shown that the total spectral flow number must be less than $N-2$, i.e., $S \leq N-2$. The same conclusion was reached from a group theoretic argument in appendix D of [16].
} 
But several details of the duality between the sigma model and Liouville theory require a more careful treatment. This applies in particular to the derivation of the shift in the background charge and to a proper regularization $\lim _{w \rightarrow z}|w-z|^{2}=-\ln |\rho(z)|^{2}$ of the divergent expression $\lim _{w \rightarrow z}|w-z|^{2}$, see [12] for details.

Through our redefinition (2.14) of the field $\phi$ we remove the factors of $\mu$ in the definition (2.2) of the vertex operators,

$$
\left|\mu_{\nu}\right|^{2\left(j_{\nu}+1\right)} e^{2 b\left(j_{\nu}+1\right) \phi\left(z_{\nu}\right)}=e^{2 b\left(j_{\nu}+1\right) \varphi\left(z_{\nu}\right)}
$$

and thereby all explicit $\mu$ dependence. It remains to rewrite the kinetic term through the new field $\varphi$. Since $\partial \bar{\partial} \phi$ and $\partial \bar{\partial} \varphi$ differ by a bunch of $\delta$-functions which are localized at the points $z_{\nu}, y_{i}$ and $\xi$, we obtain extra insertions of vertex operators $\exp \left( \pm \frac{1}{b} \varphi\right)$ at the zeroes and poles of $\mathcal{B}$. The vertex operators inserted at $z_{\nu}$ combine with the original tachyon vertex operators while those at $y_{i}$ are new. Similarly, there is an extra insertion of the operator $\exp (-S \varphi / b)$ at the point $w=\xi$. It combines with the vertex operator

$$
e^{S \phi(\xi) / b}=|u \tilde{\mathcal{B}}(\xi)|^{-S / b^{2}} e^{S \varphi(\xi) / b} \quad, \quad \tilde{\mathcal{B}}(\xi) \equiv \frac{\prod_{i=1}^{N-2-S}\left(\xi-y_{i}\right)}{\prod_{\nu=1}^{N}\left(\xi-z_{\nu}\right)}
$$

into some simple numerical factor $|u \tilde{\mathcal{B}}(\xi)|^{-S / b^{2}}$. The latter is canceled by the numerical, $\xi$-dependent term in $\phi \partial \bar{\partial} \phi$ so that the end results assume the form

$$
\begin{aligned}
& \left\langle\prod_{\nu=1}^{N} V_{j_{\nu}}\left(\mu_{\nu} \mid z_{\nu}\right) v^{S}(\xi)\right\rangle=\prod_{n=0}^{S} \delta^{2}\left(\sum_{\nu=1}^{N} \frac{\mu_{\nu}}{\left(\xi-z_{\nu}\right)^{n}}\right) \frac{\left|\Theta_{N}\right|^{2}}{|u|^{\frac{S}{b^{2}}}-2}\left\langle\prod_{\nu=1}^{N} V_{\alpha_{\nu}}\left(z_{\nu}\right) \prod_{i=1}^{N-2-S} V_{-\frac{1}{2 b}}\left(y_{i}\right)\right\rangle^{L} \\
& \text { with } \quad \Theta_{N}=\Theta_{N}\left(u, y_{j}, z_{\nu}\right)=\prod_{\mu<\nu}^{N}\left(z_{\mu \nu}\right)^{\frac{1}{2 b^{2}}} \prod_{i<j}^{N-2-S}\left(y_{i j}\right)^{\frac{1}{2 b^{2}}} \prod_{\nu=1}^{N} \prod_{i=1}^{N-2-S}\left(z_{\nu}-y_{i}\right)^{-\frac{1}{2 b^{2}}} .
\end{aligned}
$$

Note that all dependence on the insertion point $z=\xi$ has dropped from all terms but those implementing the constraints (2.12). The right hand side of eq. (2.17) is evaluated in the Liouville theory. The vertex operators are $V_{\alpha}=\exp (2 \alpha \varphi)$ with $\alpha_{\nu}=b\left(j_{\nu}+1\right)+1 / 2 b$, and the number of degenerated fields $V_{-1 / 2 b}$ is given by $N-2-S$.

All the above can be generalized to world-surfaces of higher genus $g \geq 1$, as shown in section 4, following our analysis in [12]. The main point to note concerns the number of additional insertions: On a surface of genus $g$ a one-differential with $N$ poles possesses $N+2 g-2$ zeroes. Once more, $S$ of them should come together at the point at which we insert the spectral flow of the identity field. The remaining $N+2 g-2-S$ zeros give 
rise to the insertion of degenerate fields. With the generalization to higher genus surfaces being well under control, the relation between the $H_{3}^{+}$WZNW model and Liouville field theory becomes a perturbative correspondence that works order by order in the string loop expansion.

\section{The Cigar-Sine-Liouville duality}

We are now ready to derive the duality between the cigar conformal field theory and the Sine-Liouville model. Our argument proceeds in several steps. First we use the correspondence between the $H_{3}^{+}$model and Liouville field theory to establish a similar correspondence between the cigar and a new model that involves a Liouville field $\varphi$ along with a single free boson $\chi$. As before, the Liouville correlation functions contain $N-2-S$ additional degenerate field insertions. In this form, our correspondence does not yet resemble the duality we were seeking for. To begin with, the Liouville field theory with interaction exp $2 b \varphi$ remains weakly coupled for small $b$, i.e. whenever the $H_{3}^{+}$WZNW model is weakly coupled. Furthermore, the correspondence relates correlation functions with a different number of field insertions. Finally, the background charges of the Liouville field $\varphi$ and the boson $\chi$ are found to differ from those in the Sine-Liouville model. We shall address each of these three shortfalls in a separate subsection.

\subsection{A correspondence between the cigar and Liouville theory}

Our first aim is to determine correlation functions of tachyon vertex operators in the cigar conformal field theory. As before, we parametrize the cigar geometry through the radius $R=\sqrt{k}$ of the circle at $\phi=-\infty$. The value of $R$ determines the central charge through

$$
c_{\mathrm{cig}}=2 \frac{k+1}{k-2}
$$

Let us recall that the cigar conformal field theory may be obtained from the $H_{3}^{+}$WZNW model by a process of gauging. Thereby, the cigar model gets embedded into the combination of a $H_{3}^{+}$WZNW model at level $k$ and a free bosonic field theory,

$$
S[\phi, \gamma, \beta ; X ; b, c]=S_{k}^{\mathrm{WZNW}}[\phi, \gamma, \beta]+\frac{1}{2 \pi} \int d^{2} w \partial X \bar{\partial} X+\frac{1}{2 \pi} \int d^{2} w(b \bar{\partial} c+\bar{b} \partial \bar{c})
$$

along with $(b, c)$-ghosts arising from the gauge fixing procedure (for more detail, see appendix A). The first term represents the WZNW model, written once more in a first 
order formulation (2.1). The free boson $X$ is compactified to a circle of radius $R=\sqrt{k}$, i.e. the compactification radius of $X$ is the same as for the cigar at $\phi=-\infty$. It has vanishing background charge $Q_{X}=0$.

Next we turn our attention to the vertex operators. Our conventions for vertex operators of the WZNW model can be found in (2.2). In order to spell out the relation with vertex operators of the cigar, we need to pass to the so-called $m$-basis

$$
\Phi_{m, \bar{m}}^{j}(z)=N_{m, \bar{m}}^{j} \int \frac{d^{2} \mu}{|\mu|^{2}} \mu^{m} \bar{\mu}^{\bar{m}} V_{j}(\mu \mid z), \quad N_{m, \bar{m}}^{j}=\frac{\Gamma(-j-m)}{\Gamma(j+1+\bar{m})} .
$$

We have to combine these with vertex operators of the free boson $X$. For the latter we use the following notation

$$
V_{m, \bar{m}}^{X}(z, \bar{z})=e^{i \frac{2}{\sqrt{k}}\left(m X_{L}-\bar{m} X_{R}\right)}
$$

Here, we have also introduced the fields $X_{L}=X_{L}(z, \bar{z})$ and $X_{R}=X_{R}(z, \bar{z})$ through $X=X_{L}+X_{R}$ and $\widetilde{X}=X_{L}-X_{R}$. Note that our sign convention for $\bar{m}$ deviates from the standard one. Vertex operators for the cigar are constructed according to the simple rule

$$
\Psi_{m, \bar{m}}^{j}(z, \bar{z})=V_{m, \bar{m}}^{X}(z, \bar{z}) \Phi_{m, \bar{m}}^{j}(z, \bar{z})
$$

The two parameters $m$ and $\bar{m}$ denote the left and right $\mathrm{U}(1)$ charges. They are related to the asymptotic momentum and winding numbers $n$ and $w$ (see introduction) through $m=(k w+n) / 2$ and $\bar{m}=(k w-n) / 2$.

Combining the results of the previous paragraphs, we are led to the following basic representation of our correlation functions,

$$
\begin{aligned}
\left\langle\prod_{\nu=1}^{N} \Psi_{m_{\nu}, \bar{m}_{\nu}}^{j_{\nu}}\left(z_{\nu}\right)\right\rangle^{\mathrm{cig}}= & \prod_{\nu=1}^{N}\left[N_{m_{\nu}, \bar{m}_{\nu}}^{j_{\nu}} \int \frac{d^{2} \mu_{\nu}}{\left|\mu_{\nu}\right|^{2}} \mu_{\nu}^{m_{\nu}} \bar{\mu}_{\nu}^{\bar{m}_{\nu}}\right] \times \\
& \times\left\langle V_{-\frac{k S}{2},-\frac{k S}{2}}^{X}(\xi) v^{S}(\xi) \prod_{\nu=1}^{N} V_{m_{\nu}, \bar{m}_{\nu}}^{X}\left(z_{\nu}\right) V_{j_{\nu}}\left(\mu_{\nu} \mid z_{\nu}\right)\right\rangle^{H \times F}
\end{aligned}
$$

The correlator on the right hand side is to be evaluated in a product of the $H_{3}^{+}$WZNW model with a single free boson. The parameter $S$ that determines the insertion at $z=\xi$ is related to the integers $m_{\nu}$ and $\bar{m}_{\nu}$ through $\sum_{\nu} m_{\nu}=\sum_{\nu} \bar{m}_{\nu}=\frac{k S}{2}$. For the cigar conformal field theory, the operator at $z=\xi$ is just a representation of identity field. Hence, the right hand side of eq. (3.4) should not depend on the insertion point $z=\xi$, a property we shall confirm explicitly below. 
Our first step now is to insert the results from section 2 for the correlation functions in the $H_{3}^{+}$WZNW model. Thereby, we bring in the correlators of Liouville theory, multiplied by the rather complicated prefactor $\Theta_{N}$ (see eq. (2.17)). But there remains some explicit $\mu$-dependence in the integrand along with the integration over $\mu_{\nu}$. According to our general strategy, we would like to rewrite the expressions entirely in terms of the new variables $u$ and $y_{i}$. This works out very nicely if we redefine the bosonic field $X$ in a way that is reminiscent of what we did in eq. (2.14) for the field $\phi$,

$$
\chi_{L}:=X_{L}-i \frac{\sqrt{k}}{2}\left(S \ln (w-\xi)+\sum_{i=1}^{N-2-S} \ln \left(w-y_{i}\right)-\sum_{\nu=1}^{N} \ln \left(w-z_{\nu}\right)-\ln u \rho(w)\right) .
$$

The field $\chi_{R}$ is defined by trading $X_{L}$ for $X_{R}$ and taking the complex conjugate of the second term. In this way we ensure that the local field $\chi(z, \bar{z})=\chi_{L}+\chi_{R}$ remains real. Let us also note that its dual field $\tilde{\chi}=\chi_{L}-\chi_{R}$ acquires a non-zero background charge $Q_{\tilde{\chi}}=-i \sqrt{k}$. Therefore, the free bosonic field $\chi$ has central charge $c_{\chi}=1-6 k$. Using the same reasoning as in [12] we obtain

$$
\left(\mu_{\nu}\right)^{m}\left(\bar{\mu}_{\nu}\right)^{\bar{m}} V_{m, \bar{m}}^{X}\left(z_{\nu}\right)=V_{m, \bar{m}}^{\chi}\left(z_{\nu}\right)
$$

Thereby, we now got rid of all the explicit $\mu$-dependence in the integrand. Our redefinition of the bosonic field also leads to additional insertions of bosonic vertex operators into the correlation functions, much in the same way as for the field $\varphi$.

But there is one additional important consequence of the shift (3.5). It also produces a numerical factor similar to $\Theta_{N}$, only with the exponent $1 / b^{2}$ being replaced by $-k$. Remarkably, the product of $\Theta_{N}$ with this new factor combines exactly into the Jacobian for the transformation from $\mu_{\nu}$ to $u, y_{i}$. The latter is computed in Appendix $\mathrm{C}$ and it reads

$$
\prod_{\nu=1}^{N} \frac{d^{2} \mu_{\nu}}{\left|\mu_{\nu}\right|^{2}} \prod_{n=0}^{S} \delta^{2}\left(\sum_{\nu} \frac{\mu_{\nu}}{\left(\xi-z_{\nu}\right)^{n}}\right)=\frac{\prod_{\mu<\nu}^{N}\left|z_{\mu \nu}\right|^{2} \prod_{i<j}^{N-2-S}\left|y_{i j}\right|^{2}}{\prod_{\nu=1}^{N} \prod_{i=1}^{N-2-S}\left|z_{\nu}-y_{i}\right|^{2}} \frac{d^{2} u}{|u|^{4+2 S}} \prod_{i=1}^{N-2-S} d^{2} y_{i} .
$$

In applying this substitution rule, one has to be a bit careful. Note that the parameters $\mu_{\nu}$ are not effected if we permute the insertion points $y_{i}$. This means that our transformations map the space of $\mu_{\nu}$ to a $(N-2-S)$ !-fold cover of the $y_{i}$ coordinate hyper-plane. Putting 
all this together we finally obtain

$$
\begin{aligned}
\left\langle\prod_{\nu=1}^{N} \Psi_{m_{\nu}, \bar{m}_{\nu}}^{j_{\nu}}\right\rangle^{\mathrm{cig}} & =\int \frac{\prod_{i=1}^{N-2-S} d^{2} y_{i}}{(N-2-S) !} \prod_{\nu=1}^{N} N_{m_{\nu}, \bar{m}_{\nu}}^{j_{\nu}} \times \\
& \times\left\langle\prod_{\nu=1}^{N} V_{\alpha_{\nu}}\left(z_{\nu}\right) V_{m_{\nu}-\frac{k}{2}, \bar{m}_{\nu}-\frac{k}{2}}^{\chi}\left(z_{\nu}\right) \prod_{i=1}^{N-2-S} V_{-\frac{1}{2 b}}\left(y_{i}\right) V_{\frac{k}{2}, \frac{k}{2}}^{\chi}\left(y_{i}\right)\right\rangle .
\end{aligned}
$$

The correlation function on the right hand side is evaluated in the theory

$$
S(\varphi, \chi)=\frac{1}{2 \pi} \int d^{2} w\left(\partial \varphi \bar{\partial} \varphi+\partial \chi \bar{\partial} \chi+\frac{\sqrt{g}}{4} \mathcal{R}\left(Q_{\varphi} \varphi+Q_{\tilde{\chi}} \tilde{\chi}\right)+b^{2} e^{2 b \varphi}\right)
$$

with background charges $Q_{\varphi}=b+1 / b$ and $Q_{\tilde{\chi}}=-i \sqrt{k}$. We have thereby achieved our first goal, namely to express $N$-point correlation functions in the cigar conformal field theory through correlators of $2 N-2-S$ fields in a product of the Liouville model with a single free bosonic field. This is as far as the $H_{3}^{+}$-Liouville correspondence can take us.

\subsection{Derivation of the duality with Sine-Liouville theory}

The correspondence we derived in the previous subsection falls short of being a true duality for a number of reasons. To begin with, it relates correlators in the weakly coupled cigar conformal field theory to correlation functions in weakly coupled Liouville theory. Here is where the famous self-duality of Liouville theory comes to our rescue. Through the correspondence (3.8) it provides us with a non-perturbative completion of the cigar conformal field theory, i.e. a well defined prescription to calculate cigar correlators for small values of the level $k$ (large $b$ ). The next unpleasant feature of our correspondence is that it relates correlators with a different number of vertex operators. Since the central charge of the theory (3.9) is the same as for the cigar conformal field theory, one may expect to do better. Indeed, our correspondence shall be rewritten as a duality between $N$-point correlation functions in the second subsection. At that point we could have decided to stop if we were not fully determined to recover Sine-Liouville theory. We shall succeed in the last part of this subsection through a rotation in field space and an appropriate field identification.

\subsubsection{A weak-strong coupling correspondence}

As we have just stated, the correspondence we obtained in the previous subsection does not seem very useful yet: Both the cigar and Liouville field theory get weakly coupled 
for small values of the parameter $b$ (or $k \sim 2$ ). Put differently, the Liouville interaction term $\exp (2 b \varphi)$ becomes large in the regime of small curvature radius $\sqrt{k}$ that we were hoping to describe. Our path integral manipulations could not have given us anything more. They capture the perturbative aspects of the two models and hence relate the weakly coupled regimes of the $H_{3}^{+}$(or the cigar) and Liouville field theory. What makes such a correspondence so useful is the fact that quantum Liouville theory looks the same at strong and weak coupling [17]. There is no way to derive this self-duality of Liouville field theory within the path integral treatment. But since the Liouville theory is solved (see e.g. [18] for a review and references), its self-duality under the reflection $b \rightarrow b^{-1}$ is fully established $[19,20]$.

If we are ready to accept this additional input from quantum Liouville theory, we can now compute our correlation functions in the dual theory

$$
S(\varphi, \chi)=\frac{1}{2 \pi} \int d^{2} w\left(\partial \varphi \bar{\partial} \varphi+\partial \chi \bar{\partial} \chi+\frac{\sqrt{g}}{4} \mathcal{R}\left(Q_{\varphi} \varphi+Q_{\tilde{\chi}} \tilde{\chi}\right)+\tilde{\mu} e^{\frac{2}{b} \varphi}\right)
$$

The background charge $Q_{\varphi}=b+1 / b$ of the Liouville field remains the same as before. However, the inversion of the parameter $b$ should be accompanied by an appropriate adjustment of the bulk cosmological constant. In our case, the correct choice is (see e.g. $[17,21])$

$$
\tilde{\mu}=\gamma^{-1}\left(b^{-2}\right)\left(b^{2} \gamma\left(b^{2}\right)\right)^{b^{-2}}
$$

where $\gamma(x)=\Gamma(x) / \Gamma(1-x)$, as usual. Let us stress that correlation functions of the cigar conformal field theory are still calculated through equation (3.8). There is no need to change the parameters of vertex operators, in spite of the fact that they happen to be functions of $b$. After the inversion of $b$, the interaction term $\exp 2 \varphi / b$ becomes weakly coupled when we enter the strong coupling regime of the cigar conformal field theory.

\subsubsection{Removing degenerate field insertions}

When we were discussing the correspondence between the $H_{3}^{+}$WZNW model and Liouville theory we argued that degenerate field insertions were required in order to encode all information about the target space momenta on $\mathrm{H}_{3}^{+}$. The situation has changed now. By gauging one of the directions of the $\mathrm{H}_{3}^{+}$model we descended to a 2-dimensional target space. An $N$-point function on the cigar involves only $2 N$ target space momenta and hence the $N-2$ insertion points in Liouville theory are certainly more than is needed to 
simply store information on target space momenta. Since we kept the bosonic field $X$ in our theory rather than integrating it out, it should even be possible to do without any additional field insertions.

This is indeed the case, due to the following observation [13, 22]: The integrated insertions at the points $y_{i}$ appear as if they had come from expanding an additional interaction term in the action with the field

$$
V_{-\frac{1}{2 b}}(y) V_{\frac{k}{2}, \frac{k}{2}}^{\chi}(y)=e^{-\frac{1}{b} \varphi(y, \bar{y})+i \sqrt{k} \tilde{\chi}(y, \bar{y})}
$$

where $\tilde{\chi}(y, \bar{y})=\chi_{L}-\chi_{R}$ as before. In fact, the total charge of exponentials of the field $\chi$ in our correlator is

$$
\frac{1}{\sqrt{k}} \sum_{\nu=1}^{N} m_{\nu}-N \frac{\sqrt{k}}{2}+\frac{\sqrt{k}}{2}(N-2-S)=-i Q_{\chi} .
$$

Hence, if we expand the exponential of integrated vertex operators of the form (3.12), only a single term contributes, namely the one with $N-2-S$ insertions of the interaction. Here it is essential that $\chi$ is a compact free bosonic field. Thereby, we have shown that correlation functions in the cigar conformal field theory

$$
\left\langle\prod_{\nu=1}^{N} \Psi_{m_{\nu}, \bar{m}_{\nu}}^{j_{\nu}}\right\rangle^{\mathrm{cig}}=\pi^{N-2-S} \prod_{\nu=1}^{N} N_{m_{\nu}, \bar{m}_{\nu}}^{j_{\nu}}\left\langle\prod_{\nu=1}^{N} V_{\alpha_{\nu}}\left(z_{\nu}\right) V_{m_{\nu}-\frac{k}{2}, \bar{m}_{\nu}-\frac{k}{2}}^{\chi}\left(z_{\nu}\right)\right\rangle
$$

may be computed by evaluating the correlator on the right hand side in the theory

$$
S(\varphi, \chi)=\int \frac{d^{2} w}{2 \pi}\left(\partial \varphi \bar{\partial} \varphi+\partial \chi \bar{\partial} \chi+\frac{\sqrt{g}}{4} \mathcal{R}\left(Q_{\varphi} \varphi+Q_{\tilde{\chi}} \tilde{\chi}\right)+\tilde{\mu} e^{\frac{2}{b} \varphi}-2 e^{-\frac{1}{b} \varphi+i \sqrt{k} \tilde{\chi}}\right)
$$

In our derivation, the new action $S(\varphi, \chi)$ arises as a perturbation of Liouville theory by the exponential interaction term (3.12). For the exponentiation of our degenerate field insertions into a term of the action it was crucial that we had replaced the Liouville interaction by its dual one in the previous step. In fact, only after the replacement $b \rightarrow b^{-1}$ in Liouville field theory, the two interaction terms of eq. (3.14) have a common regime in which they both become small. A functional $S(\varphi, \chi)$ with the interaction (3.12) and the original Liouville exponential $\exp (2 b \varphi)$ was considered in [23] as a possible dual of the cigar conformal field theory. The twisted Sine-Liouville theory such an $S(\varphi, \chi)$ was meant to describe, however, is not really well-defined. It certainly does not provide a weakly coupled dual for the strongly curved cigar background. 


\subsubsection{Relation with Sine-Liouville theory}

There is not much left to be done. In fact, in eqs. (3.13) and (3.14), we have derived a duality between the cigar conformal field theory and some new 2-dimensional field theory that involves two exponential interaction terms. It relates $N$ point functions between the two models and maps the strong coupling regime of one model to the weak coupling regime of the other. The only remaining problem is that our background charges and interaction terms do not look at all like those of Sine-Liouville theory.

Part of this issue can be repaired immediately. To do so, we observe that the square length $Q^{2}=Q_{\varphi}^{2}+Q_{\tilde{\chi}}^{2}$ of our background charge is the same as for Sine-Liouville theory, i.e. $Q^{2}=b^{2}$. Hence, it is possible to perform a rotation in field space from the fields $\varphi$ and $\tilde{\chi}$ to some new fields $\phi$ and $\tilde{X}$ with background charges $Q_{\phi}=b$ and $Q_{\tilde{X}}=0$, respectively, i.e.

$$
\begin{aligned}
\phi & =(k-1) \varphi-i \sqrt{k} b^{-1} \tilde{\chi}, \\
\tilde{X} & =-i \sqrt{k} b^{-1} \varphi-(k-1) \tilde{\chi} .
\end{aligned}
$$

When expressed through our new fields, the two exponential interaction terms become

$$
\begin{aligned}
& V_{L}=\exp \left(2 b^{-1} \varphi\right)=\exp \left(2 b^{-1}(k-1) \phi-2 i \sqrt{k} b^{-2} \tilde{X}\right), \\
& V_{-}=\exp \left(-b^{-1} \varphi+i \sqrt{k} \tilde{\chi}\right)=\exp \left(b^{-1} \phi-i \sqrt{k} \tilde{X}\right) .
\end{aligned}
$$

Note that the exponential $V_{-}$is one term of the tachyon potential (1.3) in Sine-Liouville theory. Only $V_{L}$ still looks very different from the second contribution $V_{+}$to the tachyon potential. But we shall see below that $V_{L}$ may be identified with $V_{+}$through a reflection with respect to the exponent of the interaction term $V_{-}$.

Before we explain the identification of $V_{L}$ and $V_{+}$we want to approach the issue of reflections in a more general context. Suppose we are given some theory $S$ with $n$ bosonic fields. We denote their background charges by $\vec{Q}=\left(Q^{i}\right)$ where $i=1, \ldots, n$. Let us also assume that the $n$ fields interact through $p$ exponential terms. These involve a sets of vectors $\vec{\beta}_{\nu}=\left(\beta_{\nu}^{i}\right)$ with $\nu$ running from $\nu=1$ to $\nu=p$. As in our example (3.17), (3.18), we shall assume $\vec{\beta}_{\nu}\left(\vec{Q}-\vec{\beta}_{\nu}\right)=1$ so that all interaction terms are marginal. With these notations introduced, our theory looks as follows,

$$
S=\frac{1}{2 \pi} \int d^{2} w\left(\sum_{i=1}^{n} \partial X_{i} \bar{\partial} X_{i}+\sum_{i=1}^{n} \frac{\sqrt{g}}{4} \mathcal{R}(\vec{Q}, \vec{X})+\sum_{\nu=1}^{p} \mu_{\nu} e^{2\left(\vec{\beta}_{\nu}, \vec{X}\right)}\right) .
$$


Now we can pass to an equivalent theory by performing one of the following reflections

$$
w_{\rho}: \vec{\beta}_{\nu} \longrightarrow \vec{\beta}_{\nu}+\vec{\beta}_{\rho}+\left(1-2\left(\vec{\beta}_{\nu}, \vec{\beta}_{\rho}\right)\right) \frac{\vec{\beta}_{\rho}}{\left(\vec{\beta}_{\rho}, \vec{\beta}_{\rho}\right)} .
$$

In other words, we can pick any pair of labels $\nu, \rho \in 1, \ldots, p$ and then replace the vectors $\beta_{\sigma}$ in our theory by

$$
\vec{\beta}_{\nu}^{\prime}=w_{\rho} \vec{\beta}_{\nu} \quad, \quad \vec{\beta}_{\sigma}^{\prime}=\vec{\beta}_{\sigma} \quad \text { for } \sigma \neq \nu .
$$

The reflection of the vector $\vec{\beta}_{\nu}$ should be accompanied by an appropriate adjustment of the corresponding bulk coupling $\mu_{\nu}$. We shall denote the corresponding coupling by $\mu_{\nu}^{\prime}$. All other bulk couplings $\mu_{\sigma}^{\prime}=\mu_{\sigma}$ with $\sigma \neq \nu$ remain the same. For $\nu=\rho$ the reflection invariance of $S$ follows from the self-duality of the Liouville field $X_{\nu}$. When $\nu \neq \rho$, the equivalence of the corresponding models is a consequence of a simple field identification (see Appendix B for more details).

Let us now apply these general remarks to the case at hand. After the rotation (3.15), (3.16), our model is of the general form (3.19) with

$$
\vec{\beta}_{1}=\left((k-1) / b,-i \sqrt{k} / b^{2}\right) \quad, \quad \vec{\beta}_{2}=(1 / 2 b,-i \sqrt{k} / 2)
$$

and $\vec{Q}=(b, 0)$. We claim that a single reflection of $\beta_{1}$ with $w_{2}$ is necessary in order to obtain the missing interaction term of the Sine-Liouville model. Indeed,

$$
w_{2}\left(\vec{\beta}_{1}\right)=(1 / 2 b, i \sqrt{k} / 2) .
$$

Hence, after reflection, our interaction term $\tilde{\mu} V_{L}$ takes the form

$$
\begin{aligned}
\tilde{\mu} V_{L}=\tilde{\mu} e^{2 \frac{k-1}{b} \phi-2 i \frac{\sqrt{k}}{b^{2}} \tilde{X}} & =-2 \pi^{2} \lambda^{2} e^{\frac{1}{b} \phi+i \sqrt{k} \tilde{X}}=-2 \pi^{2} \lambda^{2} V_{+} \\
\text {where } \quad \lambda^{2} & =\frac{\tilde{\mu}}{2 \pi^{2}} \frac{1}{\gamma(2-k)} .
\end{aligned}
$$

Here we used the reflection properties of tachyon vertex operators in $c=-2$ Liouville theory (see Appendix B). The value $c=-2$, and the precise form of the new cosmological constant, is determined by the background charge $Q_{-}=-\frac{i}{\sqrt{2}}$ of the field $-\frac{i}{\sqrt{2}}\left(b^{-1} \phi-\right.$ $i \sqrt{k} \tilde{X})$ in the exponent of $V_{-}$.

In order to make the coefficients of $V_{+}$and $V_{-}$in our final answer look more symmetrically, we shift the zero mode of $\tilde{X}$ such that we end up with

$$
S(\phi, X)=\frac{1}{2 \pi} \int d^{2} w\left(\partial \phi \bar{\partial} \phi+\partial X \bar{\partial} X+\frac{\sqrt{g}}{4} \mathcal{R} Q_{\phi} \phi+2 \pi \lambda e^{\frac{1}{b} \phi+i \sqrt{k} \tilde{X}}+2 \pi \lambda e^{\frac{1}{b} \phi-i \sqrt{k} \tilde{X}}\right) .
$$


This is indeed the action of the Sine-Liouville model. The parameter $\lambda$ is determined through $b$ by the two equations (3.22) and (3.11).

It remains to address the precise form of the vertex operators that we should use when calculating correlation functions for the cigar through Sine-Liouville theory. In equation (3.13), these took the form

$$
N_{m, \bar{m}}^{j} V_{\alpha}(z) V_{m-\frac{k}{2}, \bar{m}-\frac{k}{2}}^{\chi}(z)=\frac{\Gamma(-j-m)}{\Gamma(1+j+\bar{m})} e^{2 b\left(j+1+\frac{1}{2 b^{2}}\right) \varphi+i \frac{2}{\sqrt{k}}\left(\left(m-\frac{k}{2}\right) \chi_{L}-\left(\bar{m}-\frac{k}{2}\right) \chi_{R}\right)} .
$$

Now we rewrite the exponents of these vertex operators in terms of the rotated fields $\phi_{L}, \phi_{R}$ and $X_{L}, X_{R}$. The step requires to spit the equations (3.15), (3.16) into four equations for the left and right components of the various fields. The resulting exponents are rather complicated,

$$
\begin{aligned}
N_{m, \bar{m}}^{j} V_{\alpha}(z) V_{m-\frac{k}{2}, \bar{m}-\frac{k}{2}}^{\chi}(z) & =\frac{\Gamma(-j-m)}{\Gamma(1+j+\bar{m})} e^{2 \alpha_{\phi}^{L} \phi_{L}+2 \alpha_{X}^{L} X_{L}+2 \alpha_{\phi}^{R} \phi_{R}+2 \alpha_{X}^{R} X_{R}} \\
\text { where }\left(\begin{array}{c}
\alpha_{\phi}^{L} \\
\alpha_{X}^{L}
\end{array}\right) & =\left(\begin{array}{c}
b(k-1)\left(j+1+\frac{1}{2 b^{2}}\right)+\frac{1}{b}\left(m-\frac{k}{2}\right) \\
-i \sqrt{k}\left(j+1+\frac{1}{2 b^{2}}\right)-i \frac{1}{\sqrt{k}}(k-1)\left(m-\frac{k}{2}\right)
\end{array}\right)
\end{aligned}
$$

The parameters $\alpha_{\phi}^{R}$ and $\alpha_{X}^{R}$ are given by similar formulas but with an opposite sign in the expression for $\alpha_{X}^{R}$ and $\bar{m}$ instead of $m$. Now we perform the reflection $w_{2}^{L}$ obtained from $\beta_{2}^{L}=(1 / 2 b,-i \sqrt{k} / 2)$ on the vector $\vec{\alpha}^{L}$,

$$
w_{2}^{L}\left(\vec{\alpha}^{L}\right)=\vec{\alpha}^{L}+\vec{\beta}_{2}^{L}+\left(1-2\left(\vec{\alpha}^{L}, \vec{\beta}_{2}^{L}\right)\right) \frac{\vec{\beta}_{2}^{L}}{\left(\vec{\beta}_{2}^{L}, \vec{\beta}_{2}^{L}\right)}=(b(j+1), i m / \sqrt{k}) .
$$

The corresponding calculation for the right components differs only by some signs and results in $w_{2}^{R}\left(\vec{\alpha}^{R}\right)=(b(j+1),-i \bar{m} / \sqrt{k})$. It is remarkable that the reflection $w_{2}$ maps the complicated expression (3.25) for the vector $\vec{\alpha}$ onto something so much simpler. In particular, the reflection removed the shifts $j \rightarrow j+1 / 2 b^{2}$ and $m \rightarrow m-k / 2$ that entered our computations long ago through the redefinitions (2.14) and (3.5).

The field identification of vertex operators also involves an additional factor. This so-called reflection amplitude is worked out in Appendix B. In our case, it turns out to remove the numerical prefactor in the vertex operator (3.24), up to an overall sign. Namely, we find

$$
\begin{aligned}
N_{m, \bar{m}}^{j} V_{\alpha}(z) V_{m-\frac{k}{2}, \bar{m}-\frac{k}{2}}^{\chi}(z) & \sim-e^{2 b(j+1) \phi+i \frac{2}{\sqrt{k}}\left(m X_{L}-\bar{m} X_{R}\right)} \\
& =-e^{2 b(j+1) \phi} e^{i \frac{n}{\sqrt{k}} X+i \sqrt{k} w \widetilde{X}},
\end{aligned}
$$


where $m=(k w+n) / 2$ and $\bar{m}=(k w-n) / 2$, as before. Hence, we recovered the conventional vertex operators of Sine-Liouville theory. Inserting our results into eq. (3.13), we obtain

$$
\left\langle\prod_{\nu=1}^{N} \Psi_{m_{\nu}, \bar{m}_{\nu}}^{j_{\nu}}\right\rangle^{\mathrm{cig}}=\mathcal{N}\left\langle\prod_{\nu=1}^{N} e^{2 b\left(j_{\nu}+1\right) \phi} e^{i \frac{n_{\nu}}{\sqrt{k}} X+i \sqrt{k} w_{\nu} \widetilde{X}}\right\rangle^{\mathrm{SL}}
$$

with the overall factor $\mathcal{N}=(-1)^{N-S} \pi^{N-2-2 S} \lambda^{-S}$ depending on $\lambda$ and $S$. The right hand side of the above expression is to be evaluated in Sine-Liouville theory with radius

$R=\sqrt{k}$ and a bulk cosmological constant that is determined through $b$ by the two equations (3.22) and (3.11). Thereby, we have established the equality (1.5) of correlators in the two models on the sphere.

\section{Generalization to surfaces of higher genus}

Having successfully completed our proof of the FZZ-duality we would now like to extend it to surfaces of genus $g \geq 1$. Most of our analysis in subsection 3.2 carries over to general closed Riemann surfaces without any changes. Our main task is to extend the relation (3.8) between the cigar and Liouville field theory. In order to do so, we will briefly review our previous study [12] of $\mathrm{H}_{3}^{+}$correlation functions on higher genus surfaces. At the same time, we shall include spectral flow. As in the case of the sphere, we then descend to the cigar and derive a relation with Liouville field theory. Some necessary background material on how to gauge the $\mathrm{H}_{3}^{+}$WZNW model on higher genus surfaces is collected in Appendix A. The final step in the derivation of the correspondence between the cigar and Liouville theory requires good control of the Jacobian for the coordinate transformation from momenta $\mu_{\nu}$ etc. to insertion points $y_{i}$. This Jacobian is discussed in the technical Appendix C.

\subsection{The $H_{3}^{+}$- Liouville correspondence - genus $g \geq 1$}

From now on let $\Sigma$ be a generic Riemann surface of genus $g$ and with a fixed complex structure. On $\Sigma$ there exist $g$ holomorphic one-forms $\omega_{l}$ with $l=1, \cdots, g$. As usual, we normalize them such that

$$
\oint_{\alpha_{k}} \omega_{l}=\delta_{k l}, \quad \oint_{\beta_{k}} \omega_{l}=\tau_{k l},
$$


where the set of $\left(\alpha_{l}, \beta_{l}\right)$ is a canonical basis of homology cycles. The complex matrix $\tau$ is the period matrix of the surface $\Sigma$.

Let us turn attention to the fields $\beta, \gamma, \phi$ of the WZNW model. Originally, these are (possibly multi-valued) functions on the surface $\Sigma$. But we shall consider them as (quasi-) periodic functions on the Jacobian by means of the Abel map $\left(w_{k}\right)=\left(\int^{w} \omega_{k}\right) \in \mathbb{C}^{g}$. The periodicity conditions we impose look as follows

$$
\begin{aligned}
& \beta\left(w_{k}+\tau_{k l} n^{l}+m_{k} \mid \tau\right)=e^{2 \pi i n^{l} \lambda_{l}} \beta\left(w_{k} \mid \tau\right), \\
& \gamma\left(w_{k}+\tau_{k l} n^{l}+m_{k} \mid \tau\right)=e^{-2 \pi i n^{l} \lambda_{l}} \gamma\left(w_{k} \mid \tau\right), \\
& \phi\left(w_{k}+\tau_{k l} n^{l}+m_{k} \mid \tau\right)=\phi\left(w_{k} \mid \tau\right)+\frac{2 \pi n^{l} \operatorname{Im} \lambda_{l}}{b}
\end{aligned}
$$

for $n^{l}, m_{k} \in \mathbb{Z}$. The complex parameters $\lambda_{l}, l=1, \ldots, g$, that determine the behavior of $\beta, \gamma$ and $\phi$ under shifts along the $\beta$-cycles are called twists. Because of these twists, $\gamma$ does not possess a zero mode. On the other hand $\beta$ still has $g-1$ zero modes. These are proportional to $\lambda$-twisted holomorphic differentials $\omega_{\sigma}^{\lambda}[24,12]$.

As in the genus zero case, we compute $(N+1)$-point function in the presence of an insertion $v^{S}(\xi)$ of the spectrally flowed identity field at $z=\xi$,

$$
\left\langle\prod_{\nu=1}^{N} V_{j_{\nu}}\left(\mu_{\nu} \mid z_{\nu}\right) v^{S}(\xi)\right\rangle_{(\lambda, \varpi, \tau)}^{H}=\int \mathcal{D}^{\lambda} \phi \mathcal{D}^{\lambda} \gamma \tilde{\mathcal{D}}^{\lambda} \beta e^{-S[\phi, \gamma, \beta]} \prod_{\nu=1}^{N} V_{j_{\nu}}\left(\mu_{\nu} \mid z_{\nu}\right) e^{S \phi(\xi) / b}
$$

on a Riemann surface $\Sigma$ of genus $g$. The subscript $(\lambda, \varpi, \tau)$ indicates that we evaluate the correlator with fixed twists $\lambda_{k}$, fixed coefficients $\varpi_{\sigma}$ of the $\beta$ zero modes, and fixed complex structure $\tau_{k l}$ on the Riemann surface. The evaluation of physical correlators in the WZNW model requires setting $\lambda_{k}=0$ and integrating over zero modes $\varpi_{\sigma}$. But the construction of the correlators in the gauged model (cigar) and other applications on the WZNW model require to keep the explicit dependence on both twists and zero modes (see below).

The calculation leading from the $\mathrm{H}_{3}^{+}$WZNW model to Liouville field theory proceeds essentially as on the sphere before. It utilizes a number of rather basic functions on the Jacobian that we shall introduce while sketching the main steps of the derivation. See $[25,26,27]$ for some properties of functions on a generic Riemann surface. To begin with, we integrate out the field $\gamma(w)$, just as in section 2. Due to the presence of the various 
vertex operators $V_{j_{\nu}}\left(\mu_{\nu} \mid z_{\nu}\right)$, the field $\beta(w)$ takes the following form

$$
\beta(w)=\sum_{\nu=1}^{N} \mu_{\nu} \sigma_{\lambda}\left(w, z_{\nu}\right)+\sum_{\sigma=1}^{g-1} \varpi_{\sigma} \omega_{\sigma}^{\lambda}(w) .
$$

This expression for $\beta(w)$ replaces our formula (2.11). It involves the object $\sigma_{\lambda}\left(w, z_{\nu}\right)$ with a single pole at $w=z_{\nu}$. The latter may be constructed explicitly in terms of the theta function

$$
\theta_{\delta}(z \mid \tau)=\sum_{n \in \mathbb{Z}^{g}} \exp i \pi\left[\left(n+\delta_{1}\right)^{k} \tau_{k l}\left(n+\delta_{1}\right)^{l}+2\left(n+\delta_{1}\right)^{k}\left(z+\delta_{2}\right)_{k}\right] .
$$

Here, $\delta_{k}=\left(\delta_{1 k}, \delta_{2 k}\right)$ with $\delta_{1 k}, \delta_{2 k}=0,1 / 2$ denotes the spin structure along the homology cycles $\alpha_{k}$ and $\beta_{k}$. With the theta function $\theta_{\delta}(z \mid \tau)$ we can build the following auxiliary function $h_{\delta}(z)$ through

$$
\left(h_{\delta}(z)\right)^{2}=\sum_{k} \partial_{k} \theta_{\delta}(0 \mid \tau) \omega_{k}^{\lambda}(z)
$$

In terms of these objects, the propagator $\sigma_{\lambda}(w, z)$ can be written as $[12]$

$$
\sigma_{\lambda}(w, z)=\frac{\left(h_{\delta}(w)\right)^{2}}{\theta_{\delta}\left(\int_{z}^{w} \omega\right)} \frac{\theta_{\delta}\left(\lambda-\int_{z}^{w} \omega\right)}{\theta_{\delta}(\lambda)}
$$

with an odd spin structure $\delta$. Thereby, we have fully explained the general form (4.3) of $\beta(w)$. Next, let us see how to generalize the constraints (2.12) from the sphere to an arbitrary surface. Because we inserted the operator $v^{S}(\xi)$ in our correlator, the object $\beta(w)$ along with its first $S-1$ derivatives has to vanish at $w=\xi$,

$$
f_{n, \xi}(\mu, \varpi, \lambda):=\sum_{\nu=1}^{N} \mu_{\nu} \sigma_{\lambda}^{(n-1)}\left(\xi, z_{\nu}\right)+\sum_{\sigma=1}^{g-1} \varpi_{\sigma} \omega_{\sigma}^{\lambda(n-1)}(\xi)=0
$$

Here, the superscript $(n-1)$ stands for the $(n-1)^{t h}$ derivative and the integer $n$ runs over $n=1, \ldots, S$. In contrast to the corresponding relations (2.12), there is no constraint for $n=0$, at least as long as the twists are kept at generic values (see [12] for a more detailed discussion).

Once more, we would like to bring the function (4.3) into a product form similar to eq. (2.13). This may be achieved using another basic fact about one-differentials on a surface of genus $g$, namely that they possess $2(g-1)$ more zeros than they possess poles. Consequently, we can rewrite $\beta(w)$ as

$$
\beta(w)=u \frac{E(w, \xi)^{S} \prod_{i=1}^{M} E\left(w, y_{i}\right) \sigma(w)^{2}}{\prod_{\nu=1}^{N} E\left(w, z_{\nu}\right)} .
$$


This expression encodes the $M=N+2 g-2-S$ zeroes of order one at the points $w=y_{i}, i=1, \ldots, M$, and the zero of order $S$ at $w=\xi$. It uses the well known prime form $E(z, w)$ which is defined through

$$
E(z, w)=\frac{\theta_{\delta}\left(\int_{w}^{z} \omega \mid \tau\right)}{h_{\delta}(z) h_{\delta}(w)}
$$

where $\theta_{\delta}$ and $h_{\delta}$ are the same as in eqs. (4.4) and (4.5) above. By construction, the prime from $E(z, w)$ has a single zero at $z=w$. The other function $\sigma(w)$ that appears in the formula (4.8) is a $g / 2$-differential with neither poles nor zeros. Its definition can be found e.g. in $[12,28]$.

The rest of the calculation can be copied from our discussion in section 2. As before, we redefine the field $\phi$ through the following prescription,

$$
\begin{aligned}
\varphi(w, \bar{w}):= & \phi(w, \bar{w})+\frac{1}{2 b}\left(S \ln |E(w, \xi)|^{2}+\right. \\
& \left.+\sum_{i=1}^{M} \ln \left|E\left(w, y_{i}\right)\right|^{2}-\sum_{\nu=1}^{N} \ln \left|E\left(w, z_{\nu}\right)\right|^{2}+2 \ln |\sigma(w)|^{2}-\ln |u \rho(w)|^{2}\right) .
\end{aligned}
$$

Then we evaluate the change of the kinetic term. The resulting formula for correlation functions in the WZNW model involves Liouville correlators with $M=N+2 g-2-S$ degenerate field insertions. The precise expression is

$$
\begin{aligned}
& \left\langle\prod_{\nu=1}^{N} V_{j_{\nu}}\left(\mu_{\nu} \mid z_{\nu}\right) v^{S}(\xi)\right\rangle_{(\lambda, \varpi, \tau)}^{H}= \\
& \quad=\prod_{n=1}^{S} \delta^{2}\left(f_{n, \xi}(\mu, \varpi, \lambda)\right)|\sigma(\xi)|^{2 S}\left|\Theta_{N}^{g}\right|^{2}\left\langle\prod_{\nu=1}^{N} V_{\alpha_{\nu}}\left(z_{\nu}\right) \prod_{i=1}^{M} V_{-\frac{1}{2 b}}\left(y_{i}\right)\right\rangle_{\tau}^{L} .
\end{aligned}
$$

Our notations for fields in the Liouville correlation function on the right hand side are the same as in eq. (2.17) above. The constraint functions $f_{n, \xi}$ were introduced in eq. (4.7). In addition, the right hand side of eq. (4.11) involves a prefactor $\Theta_{N}^{g}$ of the form

$$
\begin{aligned}
\left|\Theta_{N}^{g}\right|^{2}=e^{\frac{3}{4} k U_{g}}\left|\operatorname{det}^{\prime} \nabla_{\lambda}\right|^{-2}|u|^{2-2 g-\frac{S}{b^{2}}} \prod_{\nu=1}^{N}\left|\sigma\left(z_{\nu}\right)\right|^{-2-\frac{2}{b^{2}}} \prod_{i=1}^{M}\left|\sigma\left(y_{i}\right)\right|^{2+\frac{2}{b^{2}}} \times \\
\times \prod_{\mu<\nu}^{N}\left|E\left(z_{\mu}, z_{\nu}\right)\right|^{\frac{1}{b^{2}}} \prod_{i<j}^{M}\left|E\left(y_{i}, y_{j}\right)\right|^{\frac{1}{b^{2}}} \prod_{\nu=1}^{N} \prod_{k=1}^{M}\left|E\left(z_{\nu}, y_{k}\right)\right|^{-\frac{1}{b^{2}}} .
\end{aligned}
$$

The prime in $\operatorname{det}^{\prime} \nabla_{\lambda}$ indicates that we drop the contribution from the zero mode. The function $U_{g}$, finally, is defined by

$$
U_{g}=\frac{1}{192 \pi^{2}} \int d^{2} w d^{2} y \sqrt{g(w)} \mathcal{R}(w) \sqrt{g(y)} \mathcal{R}(y) \ln |E(w, y)|^{2} .
$$


Here, $g(w)$ denotes the metric on the Riemann surface and $\mathcal{R}$ is its curvature. This concludes our derivation of the $\mathrm{H}_{3}^{+}$-Liouville correspondence for higher genus surfaces. The special case $S=0$ was treated in more detail in [12].

\subsection{The cigar-Liouville correspondence - genus $g \geq 1$}

In this subsection, we would like to relate correlators of cigar model to those of Liouville field theory with a free boson. Thereby, we shall extend eq. (3.8) to a general Riemann surface of genus $g$. Our starting point is the following presentation of the cigar correlation functions in terms of correlators of the $H_{3}^{+}$WZNW model and a free boson $X$,

$$
\left\langle\prod_{\nu=1}^{N} \Psi_{m_{\nu}, \bar{m}_{\nu}}^{j_{\nu}}\left(z_{\nu}\right)\right\rangle^{\mathrm{cig}}=\Delta_{\mathrm{FP}}(\hat{\mathcal{A}}) \int \mathcal{D} g \mathcal{D} X \prod_{l=1}^{g} d^{2} \lambda_{l} e^{-S^{\mathrm{cig}}[g, X]_{\lambda}} \prod_{\nu=1}^{N} \Psi_{m_{\nu}, \bar{m}_{\nu}}^{j_{\nu}}\left(z_{\nu}\right) .
$$

The vertex operators are given in eq. (3.3), and formula (4.14) is derived in Appendix A. The right hand side is computed in the product of an $H_{3}^{+}$WZNW model and a free bosonic field theory,

$$
\begin{aligned}
& \left\langle\prod_{\nu=1}^{N} \Psi_{m_{\nu}, \bar{m}_{\nu}}^{j_{\nu}}\left(z_{\nu}\right)\right\rangle^{\mathrm{cig}}=\left|\operatorname{det}^{\prime} \partial\right|^{2} \int \prod_{\sigma=1}^{g-1} d^{2} \varpi_{\sigma} \prod_{l=1}^{g} d^{2} \lambda_{l} \times \\
& \quad \times\left[\prod_{\nu=1}^{N} \int \frac{d^{2} \mu_{\nu}}{\left|\mu_{\nu}\right|^{2}} \mu_{\nu}^{m_{\nu}} \bar{\mu}_{\nu}^{\bar{m}_{\nu}}\right]\left\langle V_{-\frac{k S}{2},-\frac{k S}{2}}^{X}(\xi) e^{S \phi(\xi) / b} \prod_{\nu=1}^{N} V_{m_{\nu}, \bar{m}_{\nu}}^{X}\left(z_{\nu}\right) V_{j_{\nu}}\left(\mu_{\nu} \mid z_{\nu}\right)\right\rangle_{S}^{H \times F}
\end{aligned}
$$

Since the vertex operator does not include $(b, c)$-ghosts, the Faddeev-Popov determinant can be factored out. Here we have chosen the measure for $\varpi_{\sigma}$ such that the overall factor becomes simple.

Utilizing the result (4.11) from the previous subsection, we can express all $\mathrm{H}_{3}^{+}$correlators through correlation functions in the Liouville field theory. As in the case $g=0$, we redefine the field $X$ to remove the remaining explicit $\mu$-dependence,

$$
\begin{aligned}
\chi_{L}(w, \bar{w}):=X_{L} & (w, \bar{w})-i \frac{\sqrt{k}}{2}(S \ln E(w, \xi)+ \\
& \left.+\sum_{i=1}^{M} \ln E\left(w, y_{i}\right)-\sum_{\nu=1}^{N} \ln E\left(w, z_{\nu}\right)+2 \ln \sigma(w)-\ln u \rho(w)\right) .
\end{aligned}
$$

A similar redefinition is performed for $\chi_{R}$. From the definition of $X$ (see eq. (A.15) in Appendix A) we can see that $X_{L}$ and $X_{R}$ receive shifts similar to the one for the field $\phi$ when we go around a $\beta$-cycle, see the third line of eq. (4.2). Through the redefinition 
(4.16), the new fields $\chi_{L}$ and $\chi_{R}$ become periodic. The mechanism is the same as for the Liouville field $\varphi$. We can now proceed as before and obtain

$$
\begin{aligned}
\left\langle\prod_{\nu=1}^{N} \Psi_{m_{\nu}, \bar{m}_{\nu}}^{j_{\nu}}\left(z_{\nu}\right)\right\rangle^{\mathrm{cig}} & =\int \frac{\prod_{j=1}^{M} d^{2} y_{j}}{M !} \prod_{\nu=1}^{N} N_{m_{\nu}, \bar{m}_{\nu}}^{j_{\nu}} \times \\
& \times\left\langle\prod_{\nu=1}^{N} V_{\alpha_{\nu}}\left(z_{\nu}\right) V_{m_{\nu}-\frac{k}{2}, \bar{m}_{\nu}-\frac{k}{2}}^{\chi}\left(z_{\nu}\right) \prod_{j=1}^{M} V_{-\frac{1}{2 b}}\left(y_{j}\right) V_{\frac{k}{2}, \frac{k}{2}}^{\chi}\left(y_{j}\right)\right\rangle .
\end{aligned}
$$

The derivation of eq. (4.17) requires a generalization of the expression (3.7) for the Jacobian to surfaces of genus $g \geq 1$,

$$
\begin{aligned}
& \prod_{\nu=1}^{N} \frac{d^{2} \mu_{\nu}}{\left|\mu_{\nu}\right|^{2}} \prod_{\sigma=1}^{g-1} d^{2} \varpi_{\sigma} \prod_{l=1}^{g} d^{2} \lambda_{l} \prod_{n=1}^{S} \delta\left(f_{n, \xi}(\mu, \varpi, \lambda)\right) \frac{\left|\operatorname{det}^{\prime} \partial\right|^{2}}{\left|\operatorname{det}^{\prime} \nabla_{\lambda}\right|^{2}}= \\
& =\frac{\prod_{\mu<\nu}^{N}\left|E\left(z_{\mu}, z_{\nu}\right)\right|^{2} \prod_{i<j}^{M}\left|E\left(y_{i}, y_{j}\right)\right|^{2} \prod_{i=1}^{M}\left|\sigma\left(y_{i}\right)\right|^{2}}{\prod_{\nu=1}^{N} \prod_{i=1}^{M}\left|E\left(z_{\nu}, y_{i}\right)\right|^{2} \prod_{\nu=1}^{N}\left|\sigma\left(z_{\nu}\right)\right|^{2}|\sigma(\xi)|^{2 S}} \frac{d^{2} u}{|u|^{4-2 g+2 S}} \prod_{i=1}^{M} d^{2} y_{i} .
\end{aligned}
$$

We prove this formula in Appendix C. Once we have arrived at eq. (4.17), the steps we performed in section 3.2 go through without any changes. In particular, we can exponentiate the degenerate field insertions and then work our way through reflections and rotations until we arrive at the relation (1.5) between correlators on arbitrary surfaces.

\section{Conclusion and open problems}

In the previous three sections we have established complete agreement between correlation functions of tachyon vertex operators on the cigar and in Sine-Liouville theory. On the other hand, equivalence of the two models, i.e. the agreement of all correlation functions, still needs to be addressed. The proof is only completed once we have shown that both models possess the same chiral symmetry and that our tachyon vertex operators form the set of primary fields with respect to this chiral algebra. Both statements are in fact well established. Therefore, we shall only outline the main ingredients and provide a few references to the original literature.

The chiral symmetry of the cigar conformal field theory, often denoted by $\widehat{\mathcal{W}}_{\infty}(k)$, was studied many years ago, right after the model had been first discussed. A very

convincing description of $\widehat{\mathcal{W}}_{\infty}(k)$ along with extensive references to earlier contributions can be found in [29]. Given the basic fields $\phi$ and $X$ of the cigar conformal field theory 
one may construct the following parafermionic currents

$$
\Psi_{ \pm}(z)=i\left(b^{-1} \partial \phi \pm i \sqrt{k} \partial X\right) e^{ \pm 2 i \frac{1}{\sqrt{k}} X_{R}}
$$

By the equation of motion, these fields turn out to be chiral. Since their construction involves splitting the field $X$ into its chiral components, however, parafermionic currents are not local. On the other hand, $\Psi_{ \pm}(z)$ may be used to generate an infinite set $W_{s}, s=$ $2,3,4, \ldots$ of local chiral fields with weight $h_{s}=s$. Through repeated operator products one first finds the usual stress energy tensor $T=W_{2}$ and then a field $W_{3}$ of the form

$$
W_{3}(z)=\frac{6 k-8}{3}(\partial X)^{3}+\frac{2}{b^{2}}(\partial \phi)^{2} \partial X+\frac{1}{b^{3}} \partial^{2} \phi \partial X-\frac{k}{b} \partial \phi \partial^{2} X+\frac{k}{6} \partial^{3} X
$$

One may show that all higher weight fields $W_{s}, s \geq 4$, can be recovered from operator products of $T$ and $W_{3}$ alone. We can therefore think of $\widehat{\mathcal{W}}_{\infty}(k)$ either as the chiral algebra obtained from $T$ and $W_{3}$ or as the algebra of local fields with parafermionic constituents. The algebra $\widehat{\mathcal{W}}_{\infty}(k)$ suffices to generate the entire state space of the cigar conformal field theory out of the primaries $|j, m, \bar{m}\rangle=\Psi_{m, \bar{m}}^{J}(0)|0\rangle$.

Let us now switch to Sine-Liouville theory. We may employ either the explicit formula (5.1) for parafermions or the construction (5.2) of $W_{3}$ to show that the chiral symmetry algebra $\widehat{\mathcal{W}}_{\infty}(k)$ is preserved by the interaction terms in the Sine-Liouville model, i.e.

$$
\oint_{z} d w W_{3}(w) V_{ \pm}(z, \bar{z})=0=\oint_{z} d w \Psi_{\epsilon}(w) V_{ \pm}(z, \bar{z})
$$

Here, $\epsilon= \pm$ and $V_{ \pm}$are the two exponentials that appear in the interaction terms of the Sine-Liouville model. Interested readers may find a more detailed discussion and references to earlier works in [30]. In conclusion, the cigar and Sine-Liouville models possess the same chiral symmetries. Therefore, the main result of this note proves that they are equivalent.

In the introduction we have presented the AdS/CFT correspondence as our main motivation for studying the strong-weak coupling duality of the non-compact cigar geometry. Obviously, the 2-dimensional cigar is a rather simple toy model for realistic, higher dimensional holographic backgrounds, such as $A d S_{5} \times S^{5}$. Still, it is intriguing to see how the physics of a strongly curved holographic background can be mapped to a dual weakly coupled world-sheet model. Let us stress that none of the steps in our analysis seem to rely in an essential way on the particular target space dimension of the cigar. On the other 
hand, we certainly exploited the extended chiral symmetry of the model. It seems unlikely, however, that chiral symmetries are really all that crucial. In [31, 32], for example, Fateev described several dualities similar to the one between the cigar and Sine-Liouville theory, but involving massive integrable models. In any case, finding explicit higher dimensional examples of weakly coupled world-sheet models for strongly curved holographic backgrounds appears as an interesting direction for further research. Such dual models could eventually mediate between strongly coupled string physics and a weakly coupled gauge theory on the boundary of $A d S_{5}$.

Even though the extension of our analysis to higher dimensional target spaces seems possible in principle, it could be technically challenging. The space $A d S_{5} \times S^{5}$, for example, arises as a base of the coset superspace $\mathrm{SU}(2,2 \mid 4) /(\mathrm{SO}(4,1) \times \mathrm{SO}(5))$. Since (super-)groups of higher rank are involved in this construction of an $A d S_{5}$ background, the AdS/CFT correspondence motivates an extension of the correspondence between $\mathrm{H}_{3}^{+}$and Liouville theory to (super-)groups such as $\mathrm{SL}(\mathrm{N})$ or $\mathrm{PSL}(\mathrm{N} \mid \mathrm{N})$. WZNW models on $\mathrm{SL}(\mathrm{N})$, for example, possess a well studied relation with Toda field theories through Hamiltonian reduction. Encouraged by the successful treatment of SL(2), one may hope to upgrade embeddings of Toda theory into WZNW models to a full correspondence. At the critical level $k=N$, such a relation is understood as one of the ramifications of Langlands duality. Interested readers may find a detailed explanation of the so-called geometric Langlands duality and its relation to conformal field theory, along with many further references e.g. in [33] (see also $[11,34]$ for the connection with the $H_{3}^{+}$-Liouville correspondence). We hope to report on an off-critical version of the geometric Langlands duality for SL(N) and other (super-)groups of higher rank in the future.

Acknowledgements: We would like to thank Patrick Dorey, Vladimir Fateev, Amit Giveon, Sylvain Ribault, Peter Rønne, Samson Shatashvilli and Jörg Teschner for useful discussions, remarks and comments on the manuscript. The work of YH is supported in part by Research Fellowships of the Japan Society for the Promotion of Science for Young Scientists. 


\section{A Cigar field theory as a gauged WZNW model}

In this appendix we discuss the construction of the cigar conformal field theory as a gauged WZNW model, see also, e.g, [35, 36, 37, 38]. Our treatment is not restricted to the case of genus $g=0$. To begin with, let us recall that the action of the $H_{3}^{+}$WZNW model takes the form

$$
S^{\mathrm{WZNW}}[g]=\frac{k}{2 \pi} \int d^{2} w\left(\partial \phi \bar{\partial} \phi+e^{-2 \phi} \bar{\partial} \gamma \partial \bar{\gamma}\right) .
$$

Here and in the following we shall use the letter $g$ as a shorthand for the fields $\gamma, \bar{\gamma}$ and $\phi$. Upon introduction of the two auxiliary fields $\beta, \bar{\beta}$ we may recover the first order action (2.1) we have used throughout the main text, except for a different normalization of the field $\phi$.

The action of the coset theory is obtained through the usual prescription. If we decompose the $\mathrm{U}(1)$ valued gauge field $A$ through $\mathcal{A}=A d w+\bar{A} d \bar{w}$, the action of the gauged model becomes

$$
S^{\operatorname{cig}}[g, \mathcal{A}]=\frac{k}{2 \pi} \int d^{2} w\left[(\bar{\partial} \phi+\bar{A})(\partial \phi+A)+e^{-2 \phi}(\bar{\partial}+\bar{A}) \gamma(\partial+A) \bar{\gamma}\right] .
$$

Expectation values of any operator $\mathcal{O}$, such as a product of tachyon vertex operators, for example, are now computed through the associated path integral

$$
\langle\mathcal{O}\rangle^{\mathrm{cig}}=\frac{1}{V_{\mathrm{sym}}} \int \mathcal{D} g \mathcal{D} \mathcal{A} e^{-S^{\mathrm{cig}}[g, \mathcal{A}]} \mathcal{O},
$$

where $V_{\text {sym }}$ is the volume of the gauge group. Path integrals of this form may be evaluated with the help of the Faddeev-Popov prescription, by introducing an auxiliary system of $(b, c)$-ghosts. To this end insert

$$
1=\Delta_{\mathrm{FP}}(\mathcal{A}) \int\left[d x d \alpha \prod_{k=1}^{g} d^{2} \lambda_{k}\right] \delta\left(\mathcal{A}-\hat{\mathcal{A}}^{\alpha}(x, \lambda)\right)
$$

into our path integral (A.3). Here, $\Delta^{\mathrm{FP}}(\mathcal{A})$ is the Faddeev-Popov determinant and the gauge field is parametrized as

$$
\hat{\mathcal{A}}^{\alpha}(x, \lambda)=d x+* d \alpha-\pi i \bar{\lambda}_{k}\left(\tau_{2}^{-1}\right)^{k l} \omega_{l}-\pi i \lambda_{k}\left(\tau_{2}^{-1}\right)^{k l} \bar{\omega}_{l}
$$

where $k, l=1, \cdots, g$ and $\left(\tau_{2}\right)_{k l}=\operatorname{Im} \tau_{k l}$. On a sphere we can always choose $A=\partial(x+i \alpha)$ and $\bar{A}=\bar{\partial}(x-i \alpha)$. This choice is locally possible on a generic Riemann surface of genus 
$g$, but globally we have to include zero modes. These zero modes correspond to the possibly non-vanishing holonomies along the various cycles, and they are proportional to holomorphic one-form $\omega_{l}$ with $l=1, \cdots g$ on the Riemann surface. As our notation suggests, the parameter $\lambda_{k}$ turns out to be the twist along non-contractible cycles. In [12], we fixed these twists to obtain the relation between correlators of $H_{3}^{+}$model and Liouville theory. In our present context, however, we have to integrate out $\lambda_{k}$ as well. Insertion of the identity (A.4) leads to

$$
\langle\mathcal{O}\rangle^{\text {cig }}=\frac{1}{V_{\text {gauge }}} \int\left[d x d \alpha \prod_{k=1}^{g} d^{2} \lambda_{k} d g\right] \Delta_{\mathrm{FP}}\left(\hat{\mathcal{A}}^{\alpha}\right) e^{-S^{\mathrm{cig}}\left[g, \hat{\mathcal{A}}^{\alpha}\right]} \mathcal{O}
$$

after the integration over $\mathcal{A}$. Since we can show that $\Delta_{\mathrm{FP}}\left(\hat{\mathcal{A}}^{\alpha}\right)$ and $S^{\mathrm{cig}}\left[g, \hat{\mathcal{A}}^{\alpha}\right]$ are independent of $\alpha$, the integration over $\alpha$ only gives an overall factor $V_{\text {sym }}$, as long as the inserted vertex operators are independent of $\alpha$ as well.

Our first aim is to evaluate the Faddeev-Popov measure. Since the variation of gauge field is given by

$$
\delta \hat{\mathcal{A}}=d \delta x+* d \delta \alpha-\pi i \delta \bar{\lambda}_{k}\left(\tau_{2}^{-1}\right)^{k l} \omega_{l}-\pi i \delta \lambda_{k}\left(\tau_{2}^{-1}\right)^{k l} \bar{\omega}_{l}
$$

the inverse of the measure becomes

$$
\begin{aligned}
\Delta_{\mathrm{FP}}^{-1}(\hat{\mathcal{A}})= & \int\left[d \delta x d \delta \alpha \prod_{k=1}^{g} d^{2} \delta \lambda_{k}\right] \delta(\delta \hat{\mathcal{A}}) \\
= & \int\left[d^{2} \beta^{\prime} d \delta x d \delta \alpha \prod_{k=1}^{g} d^{2} \delta \lambda_{k}\right] \exp \left[2 \pi i \int d^{2} w\left(\beta^{\prime} \bar{\partial}(\delta x+i \delta \alpha)+\bar{\beta}^{\prime} \partial(\delta x-i \delta \alpha)\right)\right] \\
& \times \exp \left[2 \pi i \int d^{2} w\left(\pi \bar{\beta}_{0} \delta \bar{\lambda}_{k}\left(\tau_{2}^{-1}\right)^{k l} \omega_{l}+\pi \beta_{0} \delta \lambda_{k}\left(\tau_{2}^{-1}\right)^{k l} \bar{\omega}_{l}\right)\right] .
\end{aligned}
$$

We invert this expression following the standard trick. Thereby, we can express the Faddeev-Popov measure through an path integral over fermionic ghost systems $(b, c)$ and $(\bar{b}, \bar{c})$ along with a Grassmann integral over $2 g$ additional variables $\xi_{k}$ and $\bar{\xi}_{k}$. The latter are associated with the variations $\delta \lambda_{k}$ and $\delta \bar{\lambda}_{k}$. Thus, the measure takes the form

$$
\begin{aligned}
\Delta_{\mathrm{FP}}(\hat{\mathcal{A}}) & =\int\left[d^{2} b d^{2} c d^{2 g} \xi\right] e^{\left[-\frac{1}{2 \pi} \int d^{2} z(b \bar{\partial} c+\bar{b} \partial \bar{c})+\int\left(\bar{b}_{0} \bar{\xi}_{k}\left(\tau_{2}^{-1}\right)^{k l} \omega_{l}+b_{0} \xi_{k}\left(\tau_{2}^{-1}\right)^{k l} \bar{\omega}_{l}\right)\right]} \\
& =\int\left[d^{2} b d^{2} c\right] e^{-S_{\mathrm{gh}}[b, c]} \prod_{k=1}^{g}\left|\int \bar{b}_{0}\left(\tau_{2}^{-1}\right)^{k l} \omega_{l}\right|^{2} .
\end{aligned}
$$

In passing to the second line, we have performed the integration over $\xi_{k}$ and $\bar{\xi}_{k}$. Correlation functions are now obtained from (A.6) by inserting the expression of Faddeev-Popov 
measure (A.9). In particular, if the operator $\mathcal{O}$ does not involve any ghosts, we can explicitly perform the integration over the $(b, c)$-ghost system, which leads to

$$
\int\left[d^{2} b d^{2} c\right] e^{-S_{\mathrm{gh}}[b, c]}=\left|\operatorname{det}^{\prime} \partial\right|^{2} \operatorname{det} \tau_{2}^{-1} .
$$

One may think of the determinant $\left|\operatorname{det}^{\prime} \partial\right|^{2}$ as arising from the Jacobian $|d \mathcal{A} / d x d \alpha|$. The factor $\operatorname{det} \tau_{2}^{-1}$, on the other hand, is due to our normalization of parameter $\lambda_{k}$.

It now remains to evaluate $S^{\operatorname{cig}}\left[g, \hat{\mathcal{A}}^{\alpha=0}(x, \lambda)\right]$. Following [35], we may re-express this action through a $H_{3}^{+}$WZNW model and an additional free boson. Let us separate $x$ as $x=x_{L}+x_{R}$ with the condition $x_{L}=\left(x_{R}\right)^{*}$. Then the gauge field $A$ can be written as

$$
A=\partial x_{L}-\pi i \lambda_{k}\left(\tau_{2}^{-1}\right)^{k l} \omega_{l}=\left(\Gamma_{\lambda} e^{x_{L}}\right)^{-1} \partial\left(\Gamma_{\lambda} e^{x_{L}}\right)
$$

with

$$
\Gamma_{\lambda}(w, \bar{w})=e^{-\pi i \lambda_{k}\left(\tau_{2}^{-1}\right)^{k l}\left(\int^{w} \omega_{l}(z)-\int^{\bar{w}} \bar{\omega}_{l}(\bar{z})\right)}
$$

If the arguments are translated along the various non-trivial cycles of our surface, the factor $\Gamma_{\lambda}$ picks up the following phases

$$
\Gamma_{\lambda}\left(w_{k}+\tau_{k l} n^{l}+m_{k} \mid \tau\right)=e^{-2 \pi i n^{l} \lambda_{l}} \Gamma_{\lambda}\left(w_{k} \mid \tau\right) .
$$

Even though the anti-holomorphic part of $\Gamma_{\lambda}$ does not contribute to the chiral gauge field $A$, it is required for $\Gamma_{\lambda}$ to possess good shift properties. A similar representation can be written down for the component $\bar{A}$ of the gauge field. With this in mind we now redefine our fields according to

$$
\phi+\frac{1}{2}\left(x_{L}+x_{R}+\ln \left|\Gamma_{\lambda}\right|^{2}\right) \mapsto \phi, \quad \bar{\Gamma}_{\lambda} e^{x_{R}} \gamma \mapsto \gamma, \quad \Gamma_{\lambda} e^{x_{L}} \bar{\gamma} \mapsto \bar{\gamma}
$$

In terms of the new field, the action becomes a sum of two simple contributions,

$$
S^{\mathrm{cig}}\left[g, \mathcal{A}^{\alpha=0}(x, \lambda)\right]=S^{\mathrm{WZNW}}[g]_{\lambda}+\frac{1}{2 \pi} \int d^{2} w \bar{\partial} X \partial X
$$

The index $\lambda$ on the WZNW action indicates that the WZNW model is defined with the twist as in (4.2). Furthermore, we defined the free boson $X=X_{L}+X_{R}$ by

$$
X_{L}:=-\frac{\sqrt{k}}{2} i\left(x_{L}+\ln \Gamma_{\lambda}\right), \quad X_{R}:=\frac{\sqrt{k}}{2} i\left(x_{R}+\ln \bar{\Gamma}_{\lambda}\right) .
$$

The chiral components of $X$ also satisfy non-trivial boundary conditions, due to the shift with $\ln \Gamma_{\lambda}$. In summary, we have shown that the action for the cigar model is given by summing the action of a $\lambda$-twisted $H_{3}^{+}$WZNW model, a free boson $X$, and a $(b, c)$-system. In the main text, we only consider situations in which our insertion $\mathcal{O}$ does not involve fields $b$ and $c$. Therefore, the $(b, c)$-system decouples from the rest of the theory. 


\section{B Reflection: Self-duality and field identification}

This appendix collects a few fact about Liouville theory, its self-duality and reflection property. These are applied to multi-field models of the form (3.19) in the second subsection.

\section{B.1 Field identification in Liouville field theory}

Let us first consider a single Liouville field $\omega$ with bulk cosmological constant $\rho$ and background charge $Q_{\omega}=d+1 / d$. Our aim is to describe the reflection coefficient of fields in this theory, i.e. the function $D\left(\alpha^{L}, \alpha^{R}\right)$ that features in the relation

$$
V_{\alpha^{L}, \alpha^{R}}=D\left(\alpha^{L}, \alpha^{R}\right) V_{Q_{\omega}-\alpha^{L}, Q_{\omega}-\alpha^{R}}
$$

between vertex operators $V_{\alpha^{L}, \alpha^{R}}=\exp \left(2 \alpha^{L} \omega_{L}+2 \alpha^{R} \omega_{R}\right)$. Here, we shall allow for situations in which the exponent contains contributions from the dual Liouville field $\tilde{\omega}$, i.e. with $\alpha_{L} \neq \alpha_{R}$. The reflection coefficient $D$ is severely constrained by the operator product of generic vertex operators with degenerate ones as

$$
\begin{aligned}
& V_{-\frac{d}{2},-\frac{d}{2}}(z) V_{\alpha^{L}, \alpha^{R}}(0) \\
& \quad \sim z^{d \alpha^{L} \bar{z}^{d \alpha^{R}} V_{\alpha^{L}-\frac{d}{2}, \alpha^{L}-\frac{d}{2}}(0)+C_{-}\left(\alpha^{L}, \alpha^{R}\right) z^{d\left(Q_{\omega}-\alpha^{L}\right)} \bar{z}^{d\left(Q_{\omega}-\alpha^{R}\right)} V_{\alpha^{L}+\frac{d}{2}, \alpha^{R}+\frac{d}{2}}(0)+\ldots} .
\end{aligned}
$$

Combining this expansion with the reflection equation (B.1), we assign the following two conditions

$$
\begin{aligned}
& C_{-}\left(\alpha^{L}, \alpha^{R}\right) D\left(\alpha^{L}+\frac{d}{2}, \alpha^{R}+\frac{d}{2}\right)=D\left(\alpha^{L}, \alpha^{R}\right) \\
& D\left(\alpha^{L}, \alpha^{R}\right) D\left(Q_{\omega}-\alpha^{R}, Q_{\omega}-\alpha^{L}\right)=1 .
\end{aligned}
$$

The coefficient $C_{-}$may be determined through a free field computation, as e.g. in [39],

$$
\begin{aligned}
C_{-}\left(\alpha^{L}, \alpha^{R}\right) & =-\frac{\rho}{2 \pi} \int d^{2} x\left\langle V_{\alpha^{L}, \alpha^{R}}(0) V_{-\frac{d}{2},-\frac{d}{2}}(1) e^{2 d \omega(x)} V_{Q_{\omega}-\alpha^{L}-\frac{d}{2}, Q_{\omega}-\alpha^{R}-\frac{d}{2}}(\infty)\right\rangle \\
& =-\rho \gamma\left(1+d^{2}\right) \frac{\Gamma\left(-1+2 d \alpha^{L}-d^{2}\right) \Gamma\left(1-2 d \alpha^{R}\right)}{\Gamma\left(2-2 d \alpha^{R}+d^{2}\right) \Gamma\left(2 d \alpha^{L}\right)}
\end{aligned}
$$

There is a unique analytic solution to the constraints (B.3) that is consistent with the duality symmetry under simultaneous exchange $d \leftrightarrow 1 / d$ and $\alpha^{L} \leftrightarrow \alpha^{R}$. It is given by

$$
D\left(\alpha^{L}, \alpha^{R}\right)=\left(\rho \gamma\left(d^{2}\right)\right)^{\left(Q_{\omega}-\alpha^{L}-\alpha^{R}\right) / d} \frac{\Gamma\left(2 d \alpha^{L}-d^{2}\right) \Gamma\left(-1+\frac{2 \alpha^{R}}{d}-\frac{1}{d^{2}}\right)}{d^{2} \Gamma\left(1-2 d \alpha^{R}+d^{2}\right) \Gamma\left(2-\frac{2 \alpha^{L}}{d}+\frac{1}{d^{2}}\right)} .
$$


Applying the reflection to the Liouville field itself, i.e. to the case with $\alpha^{L}=\alpha^{R}=d$, we infer that the bulk cosmological constant $\tilde{\rho}$ of the dual Liouville field theory must take the form

$$
\tilde{\rho}=\gamma^{-1}\left(1 / d^{2}\right)\left(\rho \gamma\left(d^{2}\right)\right)^{1 / d^{2}}
$$

In the main text, the reflection of vertex operators is performed in a Liouville field theory with parameter $d=i / \sqrt{2}$ and with bulk cosmological constant $\rho=-2$. If we insert these values into our general formula for the reflection amplitude, we obtain

$$
D\left(\alpha^{L}, \alpha^{R}\right)=-\frac{\Gamma\left(1-i \sqrt{2} \alpha^{R}\right)}{\Gamma\left(i \sqrt{2} \alpha^{L}\right)} .
$$

Formulas (B.5) and (B.6) contain all the information that is needed to perform reflections of the type (3.20) we considered in section 3.2 .

\section{B.2 Reflection in theories with several bosonic fields}

Our notations and conventions in this subsection are the same as in subsection 3.2.3 of the main text. Let us suppose that $\vec{\beta}$ is one of the vectors satisfying $(\vec{\beta}, \vec{Q}-\vec{\beta})=1$. We want to analyze the field identification for a vertex operator $V_{\vec{\alpha}}$ that is induced by the Liouville interaction $\exp 2(\vec{\beta}, \vec{X})$. Here $\vec{\alpha}$ can be any vector. It is convenient to redefine

$$
X_{\vec{\beta}}=\left(d_{\vec{\beta}}^{-1} \vec{\beta}, \vec{X}\right), \quad d_{\vec{\beta}}=\sqrt{(\vec{\beta}, \vec{\beta})}
$$

The background charge for this bosonic field $X_{\vec{\beta}}$ is $q_{\vec{\beta}}=d_{\vec{\beta}}+d_{\vec{\beta}}^{-1}$ and the interaction term is now $\exp 2 d_{\vec{\beta}} X_{\vec{\beta}}$.

To begin with, let us isolate from the vector $\vec{\alpha}$ its component along $\vec{\beta}$,

$$
\vec{\alpha}=\alpha_{\vec{\beta}}\left(d_{\vec{\beta}}^{-1} \vec{\beta}\right)+\left(\vec{\alpha}-\frac{(\vec{\alpha}, \vec{\beta})}{(\vec{\beta}, \vec{\beta})} \vec{\beta}\right), \quad \alpha_{\vec{\beta}}:=d_{\vec{\beta}}^{-1}(\vec{\alpha}, \vec{\beta})
$$

The reflection along $\vec{\beta}$ is controlled by the value of the background charge $q_{\vec{\beta}}$ along $\vec{\beta}$. Hence, upon reflection, the vector $\vec{\alpha}$ gets replaced by

$$
w_{\vec{\beta}}(\vec{\alpha})=\left(q_{\vec{\beta}}-\alpha_{\vec{\beta}}\right)\left(d_{\vec{\beta}}^{-1} \vec{\beta}\right)+\left(\vec{\alpha}-\frac{(\vec{\alpha}, \vec{\beta})}{(\vec{\beta}, \vec{\beta})} \vec{\beta}\right)=\vec{\alpha}+\vec{\beta}+(1-2(\vec{\alpha}, \vec{\beta})) \frac{\vec{\beta}}{(\vec{\beta}, \vec{\beta})}
$$

Formula (B.10) is used twice in the main text, namely in eq. (3.20) and after eq. (3.25). 
The results of the previous subsection may also be used to determine the reflection amplitude that is needed to relate $V_{\vec{\alpha}}$ with its reflection $V_{w(\vec{\alpha})}$. We then find

$$
D\left(\vec{\alpha}^{L}, \vec{\alpha}^{R}\right)=\left(\mu_{\vec{\beta}} \gamma\left(d_{\vec{\beta}}^{2}\right)\right)^{\left(q_{\vec{\beta}}-\alpha_{\vec{\beta}}^{L}-\alpha_{\vec{\beta}}^{R}\right) / d_{\vec{\beta}}} \frac{\Gamma\left(2 d_{\vec{\beta}} \alpha_{\vec{\beta}}^{L}-d_{\vec{\beta}}^{2}\right) \Gamma\left(-1+\frac{2 \alpha_{\vec{\beta}}^{R}}{d_{\vec{\beta}}}-\frac{1}{d_{\vec{\beta}}^{2}}\right)}{d_{\vec{\beta}}^{2} \Gamma\left(1-2 d_{\vec{\beta}} \alpha_{\vec{\beta}}^{R}+d_{\vec{\beta}}^{2}\right) \Gamma\left(2-\frac{2 \alpha_{\vec{\beta}}^{L}}{d_{\vec{\beta}}}+\frac{1}{d_{\vec{\beta}}^{2}}\right)} .
$$

Here, $\mu_{\vec{\beta}}$ is the bulk cosmological constant in front of the interaction term $\exp 2(\vec{\beta}, \vec{X})$. In passing from an interaction term $\exp 2(\vec{\beta}, \vec{X})$ to the dual one, we must replace

$$
\vec{\beta} \longrightarrow \frac{\vec{\beta}}{(\vec{\beta}, \vec{\beta})} \quad \text { and } \quad \mu_{\vec{\beta}} \longrightarrow \tilde{\mu}_{\vec{\beta}}=\tilde{\gamma}^{-1}\left(1 / d_{\vec{\beta}}^{2}\right)\left(\mu_{\vec{\beta}} \gamma\left(d_{\vec{\beta}}^{2}\right)\right)^{1 / d_{\vec{\beta}}^{2}}
$$

The expression for the dual cosmological constant was obtained from eq. (B.6) by inserting the value $d_{\vec{\beta}}$ defined in eq. (B.8).

\section{On the Jacobian}

The aim of this appendix is to compute the Jacobian (4.18) that arises when we change variables from the momenta $\mu_{\nu}$ to insertion points $y_{i}$. We will first explain the main ideas in the case of the sphere. Thereby, we rederive eq. (3.7) that was already established in $[11,15]$. Our derivation generalizes more or less directly to surfaces of higher genus $g \geq 1$, and these will be treated in the second subsection.

\section{C.1 The Jacobian on the sphere}

For the sphere with genus $g=0$, the separation of variables (2.13) may be written in terms of the individual momenta $\mu_{\nu}$ by comparing residues,

$$
\mu_{\nu}=u \frac{\left(z_{\nu}-\xi\right)^{S} \prod_{i=1}^{N-2-S}\left(z_{\nu}-y_{i}\right)}{\prod_{\mu \neq \nu=1}^{N}\left(z_{\nu}-z_{\mu}\right)}
$$

Thereby, we obtain the following relation between differentials

$$
\frac{d \mu_{\nu}}{\mu_{\nu}}=\frac{d u}{u}-\sum_{i=1}^{N-2-S} \frac{d y_{i}}{\left(z_{\nu}-y_{i}\right)} .
$$

Before we continue, let us set $S=0$. We shall treat the more general case with $S \neq 0$ a bit later. The measure in momentum space may read

$$
\prod_{\nu=1}^{N} \frac{d^{2} \mu_{\nu}}{\left|\mu_{\nu}\right|^{2}} \delta^{2}\left(\sum_{\nu} \mu_{\nu}\right)=\prod_{\nu=1}^{N-1}\left|\frac{d u}{u}-\sum_{i=1}^{N-2} \frac{d y_{i}}{\left(z_{\nu}-y_{i}\right)}\right|^{2}\left[\frac{\prod_{\mu=1}^{N-1}\left|z_{N}-z_{\mu}\right|^{2}}{|u|^{2} \prod_{i=1}^{N-2}\left|z_{N}-y_{i}\right|^{2}}\right] .
$$


We would like to rewrite the first factor on the right hand side. In order to do so, we observe that it may be expressed through the correlation function of an auxiliary $(b, c)$ system. If we adjust the central charge such that the $b$ and $c$ possess conformal weight $h_{b}=1$ and $h_{c}=0$ and furthermore normalize the fields according to $c(z) b(y) \sim 1 /(z-y)$, we find

$$
\prod_{\nu=1}^{N-1}\left(\frac{d u}{u}-\sum_{i=1}^{N-2} \frac{d y_{i}}{\left(z_{\nu}-y_{i}\right)}\right)=\left\langle\prod_{\nu=1}^{N-1} c\left(z_{\nu}\right) \prod_{i=1}^{N-2} b\left(y_{i}\right)\right\rangle \frac{d u}{u} \prod_{i=1}^{M}\left(-d y_{i}\right) .
$$

Notice that one of the insertions $c\left(z_{\nu}\right)$ is replaced by the zero mode, i.e., a constant mode. Utilizing the usual bosonization formulas for $(b, c)$-systems we obtain

$$
\left|\left\langle\prod_{\nu=1}^{N-1} c\left(z_{\nu}\right) \prod_{i=1}^{N-2} b\left(y_{i}\right)\right\rangle\right|^{2}=\frac{\prod_{\mu<\nu=1}^{N-1}\left|z_{\mu}-z_{\nu}\right|^{2} \prod_{i<j=1}^{N-2}\left|y_{i}-y_{j}\right|^{2}}{\prod_{\nu=1}^{N-1} \prod_{i=1}^{N-2}\left|z_{\nu}-y_{i}\right|^{2}} .
$$

When this result is inserted back into eq. (C.3), we recover a special case of the Jacobian (3.7) with $S=0$,

$$
\prod_{\nu=1}^{N} \frac{d^{2} \mu_{\nu}}{\left|\mu_{\nu}\right|^{2}} \delta^{2}\left(\sum_{\nu} \mu_{\nu}\right)=\frac{\prod_{\mu<\nu=1}^{N}\left|z_{\mu}-z_{\nu}\right|^{2} \prod_{i<j=1}^{N-2}\left|y_{i}-y_{j}\right|^{2}}{\prod_{\nu=1}^{N} \prod_{i=1}^{N-2}\left|z_{\nu}-y_{i}\right|^{2}} \frac{d^{2} u}{|u|^{4}} \prod_{i=1}^{N-2} d^{2} y_{i} .
$$

In order to treat the remaining cases with $S \neq 0$, we perform an induction in $S$. So, let us assume that the relation (3.7) holds for $S=s$. If $S$ is increased to $S=s+1$, the left hand side of eq. (3.7) reads

$$
\operatorname{lhs}(3.7)=\prod_{\nu=1}^{N} \frac{d^{2} \mu_{\nu}}{\left|\mu_{\nu}\right|^{2}} \delta^{2}\left(\beta_{-s-1}\left(\mu_{\nu}\right)\right) \prod_{n=0}^{s} \delta^{2}\left(\sum_{\nu} \frac{\mu_{\nu}}{\left(\xi-z_{\nu}\right)^{n}}\right),
$$

where $\beta_{-s-1}=\sum_{\nu} \mu_{\nu}\left(\xi-z_{\nu}\right)^{-s-1}$ contains the contributions of the $N$ source terms to the mode $\beta_{-s-1}$ of $\beta$. The right hand side of eq. (3.7) can be obtained from the case $S=s$ by choosing one of the insertion points and moving it to the position $\xi$. Without loss of generality, we shall take $x:=y_{N-2-s} \rightarrow \xi$. This gives

$$
\begin{aligned}
\lim _{x \rightarrow \xi}\left(\operatorname{rhs}(3.7)_{S=s}\right)= & \frac{d^{2} u}{|u|^{4+2 s}} \prod_{i=1}^{N-3-s} d^{2} y_{i} d^{2} x \delta^{2}\left(\beta_{-s-1}\left(y_{i}, x\right)\right) \times \\
& \times \frac{\prod_{\mu<\nu}^{N}\left|z_{\mu}-z_{\nu}\right|^{2} \prod_{i<j}^{N-3-s}\left|y_{i}-y_{j}\right|^{2} \prod_{i=1}^{N-3-s}\left|y_{i}-x\right|^{2}}{\prod_{\nu=1}^{N} \prod_{i=1}^{N-3-s}\left|z_{\nu}-y_{i}\right|^{2} \prod_{\nu=1}^{N}\left|z_{\nu}-x\right|^{2}}
\end{aligned}
$$

Thereby, we have reduced our problem to showing that

$$
\delta^{2}\left(\beta_{-s-1}\left(y_{j}, x\right)\right)=\frac{1}{|u|^{2}} \frac{\prod_{\nu=1}^{N}\left|z_{\nu}-\xi\right|^{2}}{\prod_{i=1}^{N-3-s}\left|y_{i}-\xi\right|^{2}} \delta^{2}(x-\xi) .
$$


For $S=s$, the function $\beta(w)$ is known to take the form

$$
\beta(w)=u \frac{(w-\xi)^{s}(w-x) \prod_{i=1}^{N-3-s}\left(w-y_{i}\right)}{\prod_{\nu=1}^{N}\left(w-z_{\nu}\right)} .
$$

We can take this expression and expand around $x \sim \xi$ to obtain the following expression for the mode $\beta_{-s-1}$

$$
\beta_{-s-1}=u \frac{\prod_{i=1}^{N-3-s}\left(\xi-y_{i}\right)}{\prod_{\nu=1}^{N}\left(\xi-z_{\nu}\right)}(\xi-x)
$$

in terms of $y_{i}$. This equation leads to eq. (C.8), and thereby establishes that the formula eq. (3.7) for the Jacobian is valid for all $0 \leq S \leq N-2$.

\section{C.2 The Jacobian for genus $g \geq 1$}

We now repeat the steps of the previous subsection in the case of generic genus $g \geq 1$. In this case, the separation of variables takes the form

$$
\sum_{\nu=1}^{N} \mu_{\nu} \sigma_{\lambda}\left(w, z_{\nu}\right)+\sum_{\sigma=1}^{g-1} \varpi_{\sigma} \omega_{\sigma}^{\lambda}(w)=u \frac{E(w, \xi)^{S} \prod_{i=1}^{M} E\left(w, y_{i}\right) \sigma(w)^{2}}{\prod_{\nu=1}^{N} E\left(w, z_{\nu}\right)} .
$$

Here we have denoted $M=N-2 g-2-S$, as before. From this equation we can deduce the formula for the momenta $\mu_{\nu}$ as

$$
\mu_{\nu}=u \frac{E\left(z_{\nu}, \xi\right)^{S} \prod_{i=1}^{M} E\left(z_{\nu}, y_{i}\right) \sigma\left(z_{\nu}\right)^{2}}{\prod_{\mu \neq \nu}^{N} E\left(z_{\nu}, z_{\mu}\right)}, \quad \frac{d \mu_{\nu}}{\mu_{\nu}}=\frac{d u}{u}+\sum_{i=1}^{M} \partial_{y_{i}} \ln E\left(z_{\nu}, y_{i}\right) d y_{i} .
$$

This expression strongly indicates that the Jacobian could be derived with the use of $(b, c)$-ghost system since the propagator of a $(b, c)$-system on a surface of genus $g$ can be expressed through the prime form as $\langle c(z) b(y)\rangle=\partial_{y} \ln E(z, y)$.

In the case of genus zero only the integral over the momenta $\mu_{\nu}$ appears in the left hand side of (3.7), but in the case of generic genus the integrals over the twists $\lambda_{l}$ and over the zero modes $\varpi_{\sigma}$ are involved in (4.18) as well. For the twists $\lambda_{l}$ we utilize the relations to $y_{i}$ as

$$
\lambda_{l}=S \int_{w}^{\xi} \omega_{l}+\sum_{i=1}^{M} \int_{w}^{y_{i}} \omega_{l}-\sum_{\nu=1}^{N} \int_{w}^{z_{\nu}} \omega_{l}-2 \int_{(g-1) w}^{\Delta} \omega_{l}
$$

where $\Delta$ denotes the Riemann constant. By acting with the differential $d$ on these equations, we obtain the following simple relations as

$$
d \lambda_{l}=\sum_{i=1}^{M} \omega_{l}\left(y_{i}\right) d y_{i}
$$


For the zero modes $\varpi_{\sigma}$ we use the general expression as

$$
\begin{aligned}
& \sum_{\nu=1}^{N} d \mu_{\nu} \sigma_{\lambda}\left(\eta_{\rho}, z_{\nu}\right)+\sum_{\sigma=1}^{g-1} d \varpi_{\sigma} \omega_{\sigma}^{\lambda}\left(\eta_{\rho}\right) \\
& =u \frac{E\left(\eta_{\rho}, \xi\right)^{S} \prod_{i=1}^{M} E\left(\eta_{\rho}, y_{i}\right) \sigma\left(\eta_{\rho}\right)^{2}}{\prod_{\nu=1}^{N} E\left(\eta_{\rho}, z_{\nu}\right)}\left[\frac{d u}{u}+\sum_{i} \partial_{y_{i}} E\left(\eta_{\rho}, y_{i}\right) d y_{i}\right],
\end{aligned}
$$

which is deduced from eq. (C.11). Here we set $w=\eta_{\rho}$ as an arbitrary point on the Riemann surface $\Sigma$.

As in the previous subsection we start from $S=0$ case and then generalize to $S \neq 0$ case by making use of the induction procedure. One may worry about the measure of zero modes as the expression in the left hand side of (C.14) involves $d \mu_{\nu}$ in addition to $d \varpi_{\sigma}$. This problem can be resolved by the following simple observation,

$$
\begin{gathered}
\prod_{\nu=1}^{N} \frac{d \mu_{\nu}}{\mu_{\nu}} \prod_{\rho=1}^{g-1}\left[\sum_{\nu=1}^{N} d \mu_{\nu} \sigma_{\lambda}\left(\eta_{\rho}, z_{\nu}\right)+\sum_{\sigma=1}^{g-1} d \varpi_{\sigma} \omega_{\sigma}^{\lambda}\left(\eta_{\rho}\right)\right] \prod_{l=1}^{g} d \lambda_{l}= \\
=\prod_{\nu=1}^{N} \frac{d \mu_{\nu}}{\mu_{\nu}} \prod_{\sigma=1}^{g-1} d \varpi_{\sigma} \prod_{l=1}^{g} d \lambda_{l} \operatorname{det}_{\sigma, \rho} \omega_{\sigma}^{\lambda}\left(\eta_{\rho}\right) .
\end{gathered}
$$

Combining everything obtained above, the left hand side of (4.18) can be rewritten as

$$
\begin{aligned}
& \prod_{\nu=1}^{N} \frac{d \mu_{\nu}}{\mu_{\nu}} \prod_{\sigma=1}^{g-1} d \varpi_{\sigma} \prod_{l=1}^{g} d \lambda_{l}=\frac{1}{\operatorname{det}_{\sigma, \rho} \omega_{\sigma}^{\lambda}\left(\eta_{\rho}\right)} \prod_{\nu=1}^{N}\left[\frac{d u}{u}+\sum_{i=1}^{M} \partial_{y_{i}} \ln E\left(z_{\nu}, y_{i}\right) d y_{i}\right] \\
& \times \prod_{\rho=1}^{g-1}\left\{u \frac{\prod_{i=1}^{M} E\left(\eta_{\rho}, y_{i}\right) \sigma\left(\eta_{\rho}\right)^{2}}{\prod_{\nu=1}^{N} E\left(\eta_{\rho}, z_{\nu}\right)}\left[\frac{d u}{u}+\sum_{i=1}^{M} \partial_{y_{i}} \ln E\left(\eta_{\rho}, y_{i}\right) d y_{i}\right]\right\} \prod_{l=1}^{g}\left[\sum_{i=1}^{M} \omega_{l}\left(y_{i}\right) d y_{i}\right] .
\end{aligned}
$$

Once again it is advantageous to express the right hand side of the previous equality through correlators in an auxiliary $(b, c)$-system. Notice that $b(y)$ has $g$ zero modes $\omega_{l}(y)$ for genus $g$. Moreover, there is a single constant mode for $c(w)$ (see, e.g., [28]). With these facts and the propagator of $(b, c)$-system, we can express the measure as

$$
\begin{aligned}
& \prod_{\nu=1}^{N} \frac{d^{2} \mu_{\nu}}{\left|\mu_{\nu}\right|^{2}} \prod_{\sigma=1}^{g-1} d^{2} \varpi_{\sigma} \prod_{l=1}^{g} d^{2} \lambda_{l}=\frac{1}{\left|\operatorname{det}_{\sigma, \rho} \omega_{\sigma}^{\lambda}\left(\eta_{\rho}\right)\right|^{2}} \frac{1}{\left|\operatorname{det}^{\prime} \partial\right|^{2}} \times \\
& \quad \times \prod_{\rho=1}^{g-1}\left\{\frac{\prod_{i=1}^{M}\left|E\left(\eta_{\rho}, y_{i}\right)\right|^{2}\left|\sigma\left(\eta_{\rho}\right)\right|^{4}}{\prod_{\nu=1}^{N}\left|E\left(\eta_{\rho}, z_{\nu}\right)\right|^{2}}\right\}\left|\left\langle\prod_{\sigma=1}^{g-1} c\left(\eta_{\sigma}\right) \prod_{\nu=1}^{N} c\left(z_{\nu}\right) \prod_{k=1}^{M} b\left(y_{k}\right)\right\rangle\right|^{2} \frac{d^{2} u}{|u|^{4-2 g}} \prod_{i=1}^{M} d^{2} y_{i} .
\end{aligned}
$$

The factor $1 /\left|\operatorname{det}^{\prime} \partial\right|^{2}$ is included to divide the contribution from the partition function. 
In the following we will show that eq. (C.17) is indeed equal to eq. (4.18) by utilizing the bosonization formulas of $(b, c)$-systems. First, we rewrite the correlation function of $(b, c)$-ghosts in $(\mathrm{C} .17)$ as $[28]$

$$
\begin{aligned}
& \left|\left\langle\prod_{\sigma=1}^{g-1} c\left(\eta_{\sigma}\right) \prod_{\nu=1}^{N} c\left(z_{\nu}\right) \prod_{k=1}^{M} b\left(y_{k}\right)\right\rangle\right|^{2}=\frac{1}{\left|\operatorname{det}^{\prime} \partial\right|}\left|\theta\left(\sum_{\sigma} \eta_{\sigma}+\sum_{\nu} z_{\nu}-\sum_{i} y_{i}+\Delta\right)\right|^{2} \times \quad \text { (C.18) } \\
& \times \frac{\prod_{\sigma<\rho}^{g-1}\left|E\left(\eta_{\sigma}, \eta_{\rho}\right)\right|^{2} \prod_{\sigma=1}^{g-1} \prod_{\nu=1}^{N}\left|E\left(\eta_{\sigma}, z_{\nu}\right)\right|^{2} \prod_{\mu<\nu}^{N}\left|E\left(z_{\mu}, z_{\nu}\right)\right|^{2} \prod_{i<j}^{M}\left|E\left(y_{i}, y_{j}\right)\right|^{2} \prod_{i=1}^{M}\left|\sigma\left(y_{i}\right)\right|^{2}}{\prod_{\sigma=1}^{g-1} \prod_{i=1}^{M}\left|E\left(\eta_{\sigma}, y_{i}\right)\right|^{2} \prod_{\nu=1}^{N} \prod_{i=1}^{M}\left|E\left(z_{\nu}, y_{i}\right)\right|^{2} \prod_{\rho=1}^{g-1}\left|\sigma\left(\eta_{\rho}\right)\right|^{2} \prod_{\nu=1}^{N}\left|\sigma\left(z_{\nu}\right)\right|^{2}} .
\end{aligned}
$$

The factor $\left(\operatorname{det}^{\prime} \partial \bar{\partial}\right)^{-1 / 2}$ is the partition function of a complex boson. The theta function, which may be written as $\left|\theta\left(\sum_{\sigma} \eta_{\sigma}-\Delta-\lambda\right)\right|$ by means of eq. (C.12), arises from summing over the solitonic modes. Notice that the factors involving $E\left(\eta_{\sigma}, z_{\nu}\right)$ and $E\left(\eta_{\sigma}, y_{i}\right)$ are canceled if we insert the above expression (C.18) into eq. (C.17). In this way, the entire dependence on $\eta_{\sigma}$ resides in a single factor that is independent on any of the variables. Since the $\eta_{\sigma}$-dependence is expected to drop out in the end, the cancellation of terms involving both $y_{i}, z_{\nu}$ and $\eta_{\sigma}$ is an important intermediate step.

In order to incorporate the last factor on the left hand side of our formula (4.18), we need to analyze the partition function with twists $\lambda_{k}$. Up to now we worked with a $(b, c)$-ghost system without twists, but this does not yield any partition function with $\lambda$-dependence. Therefore, we shall now deal with $(b, c)$-ghosts with twists $\lambda_{k}$, where the ghosts satisfy the same twisted boundary conditions as the $(\beta, \gamma)$-system. Consequently, the $b$-ghost has $g-1$ zero modes which are proportional to the $g-1$ twisted holomorphic one-differentials $\omega_{\sigma}^{\lambda}(w)$. The simplest non-zero correlator in the twisted $(b, c)$-system is

$$
\left\langle\prod_{\rho=1}^{g-1} b\left(\eta_{\rho}\right)\right\rangle_{\lambda}=\operatorname{det}^{\prime} \nabla_{\lambda} \operatorname{det}_{\sigma, \rho} \omega_{\sigma}^{\lambda}\left(\eta_{\rho}\right)
$$

We show now that this function is useful to remove the $\eta_{\sigma}$-dependence in eq. (C.17). Again application of the usual bosonization formulas leads to

$$
\left|\left\langle\prod_{\rho=1}^{g-1} b\left(\eta_{\rho}\right)\right\rangle\right|^{2}=\frac{1}{\left|\operatorname{det}^{\prime} \partial\right|}\left|\theta\left(\sum_{\sigma} \eta_{\sigma}-\Delta-\lambda\right)\right|^{2} \prod_{\sigma<\rho=1}^{g-1}\left|E\left(\eta_{\sigma}, \eta_{\rho}\right)\right|^{2} \prod_{\sigma=1}^{g-1}\left|\sigma\left(\eta_{\sigma}\right)\right|^{2} .
$$

Then the combination with eq. (C.19) gives the equality

$$
\left|\operatorname{det}^{\prime} \nabla_{\lambda}\right|^{2}=\frac{1}{\left|\operatorname{det}_{\sigma, \rho} \omega_{\sigma}^{\lambda}\left(\eta_{\rho}\right)\right|^{2}} \frac{1}{\left|\operatorname{det}^{\prime} \partial\right|}\left|\theta\left(\sum_{\sigma} \eta_{\sigma}-\Delta-\lambda\right)\right|^{2} \prod_{\sigma<\rho=1}^{g-1}\left|E\left(\eta_{\sigma}, \eta_{\rho}\right)\right|^{2} \prod_{\sigma=1}^{g-1}\left|\sigma\left(\eta_{\sigma}\right)\right|^{2} .
$$


This equality removes the all $\eta_{\sigma}$-dependent terms and at the same time leads to eq. (4.18) for $S=0$.

The cases with $S \neq 0$ are treated as in the previous subsection, i.e. by induction in $S$. Therefore, we assume that the Jacobian is of the anticipated form when $S=s$ and try to establish the same for $S=s+1$. The first few steps are performed in precisely the same way as on the sphere. They lead to the following requirement

$$
\delta^{2}\left(\beta_{-s-1}\left(y_{i}, x\right)\right)=\frac{1}{|u|^{2}} \frac{\prod_{\nu=1}^{N}\left|E\left(\xi, z_{\nu}\right)\right|^{2}}{\prod_{i=1}^{N-2 g-3-s}\left|E\left(\xi, y_{i},\right)\right|^{2}|\sigma(\xi)|^{4}} \delta^{2}(x-\xi)
$$

that replaces our formula (C.8) from the previous subsection. We may prove this equation by recalling that the prime form behaves as $E(\xi, x) \sim \xi-x$ for $\xi \sim x$. Therefore, the mode expansion of $\beta(w)$ around $w \sim \xi$ gives

$$
\beta_{-s-1}=u \frac{\prod_{i=1}^{N-2 g-2-s} E\left(\xi, y_{i}\right) \sigma(\xi)^{2}}{\prod_{\nu=1}^{N} E\left(\xi, z_{\nu}\right)}(\xi-x) .
$$

With this result we can easily deduce first eq. (C.21) and then the anticipated expression (4.18) for the Jacobian with $S=s+1$ from the case $S=s$. Thereby, we conclude our derivation of the Jacobian (4.18).

\section{References}

[1] A. Giveon, M. Porrati and E. Rabinovici, "Target space duality in string theory," Phys. Rept. 244, 77 (1994) [arXiv:hep-th/9401139].

[2] S. Elitzur, A. Forge and E. Rabinovici, "Some global aspects of string compactifications," Nucl. Phys. B 359, 581 (1991).

[3] G. Mandal, A. M. Sengupta and S. R. Wadia, "Classical solutions of two-dimensional string theory," Mod. Phys. Lett. A 6, 1685 (1991).

[4] E. Witten, "On string theory and black holes," Phys. Rev. D 44, 314 (1991).

[5] V. Kazakov, I. K. Kostov and D. Kutasov, "A matrix model for the two-dimensional black hole," Nucl. Phys. B 622, 141 (2002) [arXiv:hep-th/0101011].

[6] V.A. Fateev, A.B. Zamolodchikov and Al.B. Zamolodchikov, unpublished. 
[7] T. H. Buscher, "A symmetry of the string background field equations," Phys. Lett. B 194, 59 (1987).

[8] T. H. Buscher, "Path integral derivation of quantum duality in nonlinear sigma models," Phys. Lett. B 201, 466 (1988).

[9] T. Fukuda and K. Hosomichi, "Three-point functions in Sine-Liouville theory," JHEP 0109, 003 (2001) [arXiv:hep-th/0105217].

[10] K. Hori and A. Kapustin, "Duality of the fermionic $2 \mathrm{~d}$ black hole and $\mathcal{N}=2$ Liouville theory as mirror symmetry," JHEP 0108, 045 (2001) [arXiv:hep-th/0104202].

[11] S. Ribault and J. Teschner, " $H_{3}^{+}$WZNW correlators from Liouville theory," JHEP 0506, 014 (2005) [arXiv:hep-th/0502048].

[12] Y. Hikida and V. Schomerus, " $H_{3}^{+}$WZNW model from Liouville field theory," JHEP 0710, 064 (2007) [arXiv:0706.1030 [hep-th]].

[13] V. Fateev, unpublished.

[14] J. M. Maldacena and H. Ooguri, "Strings in $A d S_{3}$ and $\operatorname{SL}(2, \mathbb{R})$ WZW model. I: The spectrum," J. Math. Phys. 42, 2929 (2001) [arXiv:hep-th/0001053].

[15] S. Ribault, "Knizhnik-Zamolodchikov equations and spectral flow in $A d S_{3}$ string theory," JHEP 0509, 045 (2005) [arXiv:hep-th/0507114].

[16] J. M. Maldacena and H. Ooguri, "Strings in $A d S_{3}$ and the $\mathrm{SL}(2, \mathbb{R})$ WZW model. III: Correlation functions," Phys. Rev. D 65, 106006 (2002) [arXiv:hep-th/0111180].

[17] A. B. Zamolodchikov and A. B. Zamolodchikov, "Structure constants and conformal bootstrap in Liouville field theory," Nucl. Phys. B 477, 577 (1996) [arXiv:hepth/9506136].

[18] V. Schomerus, "Non-compact string backgrounds and non-rational CFT," Phys. Rept. 431, 39 (2006) [arXiv:hep-th/0509155].

[19] B. Ponsot and J. Teschner, "Liouville bootstrap via harmonic analysis on a noncompact quantum group," arXiv:hep-th/9911110. 
[20] J. Teschner, "A lecture on the Liouville vertex operators," Int. J. Mod. Phys. A 19S2, 436 (2004) [arXiv:hep-th/0303150].

[21] J. Teschner, "Liouville theory revisited," Class. Quant. Grav. 18, R153 (2001) [arXiv:hep-th/0104158].

[22] G. Giribet, "The string theory on $A d S_{3}$ as a marginal deformation of a linear dilaton background," Nucl. Phys. B 737, 209 (2006) [arXiv:hep-th/0511252].

[23] G. Giribet and M. Leoni, "A twisted FZZ-like dual for the 2D black hole," arXiv:0706.0036 [hep-th].

[24] D. Bernard, "On the Wess-Zumino-Witten models on Riemann surfaces," Nucl. Phys. B 309, 145 (1988).

[25] J. Fay, "Theta functions on Riemann surfaces," Lecture Notes in Mathematics 352, Springer-Verlag (1973).

[26] D. Mumford, "Tata lectures on theta, Vols. I, II," Progress in Mathematics 43, Birkhäuser (1984)

[27] L. Alvarez-Gaume, G. W. Moore and C. Vafa, "Theta functions, modular invariance, and strings," Commun. Math. Phys. 106, 1 (1986).

[28] E. P. Verlinde and H. L. Verlinde, "Chiral bosonization, determinants and the string partition function," Nucl. Phys. B 288, 357 (1987).

[29] I. Bakas and E. Kiritsis, "Beyond the large $N$ limit: Non-linear $W_{\infty}$ as symmetry of the $\mathrm{SL}(2, \mathbb{R}) / \mathrm{U}(1)$ coset model," Int. J. Mod. Phys. A 7S1A, 55 (1992) [Int. J. Mod. Phys. A 7, 55 (1992)] [arXiv:hep-th/9109029].

[30] V. A. Fateev and S. L. Lukyanov, "Boundary RG flow associated with the AKNS soliton hierarchy," J. Phys. A 39, 12889 (2006) [arXiv:hep-th/0510271].

[31] V. A. Fateev, "The duality between two-dimensional integrable field theories and sigma models," Phys. Lett. B 357, 395 (1995).

[32] V. A. Fateev, "The sigma model (dual) representation for a two-parameter family of integrable quantum field theories," Nucl. Phys. B 473, 509 (1996). 
[33] E. Frenkel, "Lectures on the Langlands program and conformal field theory," arXiv:hep-th/0512172.

[34] G. Giribet, Y. Nakayama and L. Nicolas, "Langlands duality in Liouville- $H_{3}^{+}$WZNW correspondence," arXiv:0805.1254 [hep-th].

[35] K. Gawedzki and A. Kupiainen, "Coset construction from functional integrals," Nucl. Phys. B 320, 625 (1989).

[36] K. Gawedzki, "Non-compact WZW conformal field theories," arXiv:hep-th/9110076.

[37] E. J. Martinec and S. L. Shatashvili, "Black hole physics and Liouville theory," Nucl. Phys. B 368 (1992) 338.

[38] R. Dijkgraaf, H. L. Verlinde and E. P. Verlinde, "String propagation in a black hole geometry," Nucl. Phys. B 371, 269 (1992).

[39] V. S. Dotsenko, "Lectures on conformal field theory," Advanced Studies in Pure Mathematics 16, 123 (1988). 